\title{
EXISTENCE AND CONCENTRATION OF SEMI-CLASSICAL SOLUTIONS FOR DIRAC EQUATIONS WITH CRITICAL NONLINEARITIES
}

\author{
YANHENG DING* AND BERNHARD RUF ${ }^{\dagger}$
}

\begin{abstract}
We study the semi-classical ground states of the Dirac equation with critical nonlinearity:

$$
-i \hbar \alpha \cdot \nabla w+a \beta w+V(x) w=W(x)(g(|w|)+|w|) w
$$

for $x \in \mathbb{R}^{3}$. The Dirac operator is unbounded from below and above so the associate energy functional is strongly indefinite. We develop an argument to establish the existence of least energy solutions for $\hbar$ small. We also describe the concentration phenomena of the solutions as $\hbar \rightarrow 0$.
\end{abstract}

Key words. nonlinear Dirac equation, semiclassical states, concentration

AMS subject classifications. 35Q40, 49J35

1. Introduction. This paper aims to study the existence and concentration phenomena of semiclassical ground states for the stationary Dirac equation with critical nonlinearities:

$$
-i \hbar \sum_{k=1}^{3} \alpha_{k} \partial_{k} w+a \beta w+V(x) w=W(x)(g(|w|)+|w|) w
$$

with $w: \mathbb{R}^{3} \rightarrow \mathbb{C}^{4}$. Equation (1.1) is a first order partial differential equation on $\mathbb{R}^{3}$; the relevant Sobolev embedding is $H^{1 / 2}\left(\mathbb{R}^{3}, \mathbb{C}^{4}\right) \subset L^{3}\left(\mathbb{R}^{3}, \mathbb{C}^{4}\right)$, i.e. the Sobolev critical growth is 3 . Thus the term $|w| w$ has critical growth, while $g(|w|) w$ is assumed to be superlinear and subcritical as $|w| \rightarrow \infty$. In (1.1), $\hbar$ denotes Plank's constant, $\partial_{k}=\frac{\partial}{\partial x_{k}}, a>0$ is a constant, $\alpha_{1}, \alpha_{2}, \alpha_{3}$ and $\beta$ are $4 \times 4$ complex matrices:

$$
\beta=\left(\begin{array}{cc}
I & 0 \\
0 & -I
\end{array}\right), \quad \alpha_{k}=\left(\begin{array}{cc}
0 & \sigma_{k} \\
\sigma_{k} & 0
\end{array}\right), \quad k=1,2,3
$$

with

$$
\sigma_{1}=\left(\begin{array}{cc}
0 & 1 \\
1 & 0
\end{array}\right), \quad \sigma_{2}=\left(\begin{array}{cc}
0 & -i \\
i & 0
\end{array}\right), \quad \sigma_{3}=\left(\begin{array}{cc}
1 & 0 \\
0 & -1
\end{array}\right)
$$

and $V, W: \mathbb{R}^{3} \rightarrow \mathbb{R}$ are continuous functions. In the sequel, for notational convenience, we will write $\alpha=\left(\alpha_{1}, \alpha_{2}, \alpha_{3}\right)$ and $\alpha \cdot \nabla=\sum_{k=1}^{3} \alpha_{k} \partial_{k}$, as well as

$$
\begin{aligned}
& \tau:=\min V, \quad \mathscr{V}:=\left\{x \in \mathbb{R}^{3}: V(x)=\tau\right\}, \\
& \tau_{\infty}:=\liminf _{|x| \rightarrow \infty} V(x), \\
& \pi:=\max W, \quad \mathscr{W}:=\left\{x \in \mathbb{R}^{3}: W(x)=\pi\right\}, \\
& \pi_{\infty}:=\limsup _{|x| \rightarrow \infty} W(x) .
\end{aligned}
$$

*Institute of Mathematics, AMSS, Chinese Academy of Sciences, 100190 Beijing, China, and International Center for Theoretical Physics, Trieste, Italy (dingyh@math.ac.cn). Y. Ding was supported by the National Science Foundation of China (NSFC10831005, 10721061, 11171286).

${ }^{\dagger}$ Dip. di Matematica, University of Milan, 20133 Milano, Italy (bernhard.ruf@unimi.it) 
Our arguments depend crucially on these numbers and sets.

Equation (1.1) or the more general one

$$
-i \hbar \alpha \cdot \nabla w+a \beta w+M(x) w=F_{w}(x, w),
$$

arises when one seeks standing wave solutions of the nonlinear Dirac equation

$$
-i \hbar \partial_{t} \psi=i c \hbar \alpha \cdot \nabla \psi-m c^{2} \beta \psi-V(x) \psi+G_{\psi}(x, \psi) .
$$

Such equations have been widely used to build relativistic models of extended particles by means of nonlinear Dirac fields. Different functions $G$ model various types of selfcouplings [25]. Assuming that $G\left(x, e^{i \theta} \psi\right)=G(x, \psi)$ for all $\theta \in[0,2 \pi]$, a standing wave solution of (1.3) is a solution of the form $\psi(t, x)=e^{\frac{i \mu t}{\hbar}} w(x)$. It is clear that $\psi(t, x)$ solves (1.3) if and only if $w(x)$ solves (1.2) with $a=m c, M(x)=V(x) / c+\mu I_{4}$ and $F(x, w)=G(x, w) / c$.

Several papers have been devoted to the study of the existence of solutions of (1.2) under various hypotheses on the potential functions and the nonlinearity (see [18] for a review). In [5] the authors studied the problem with $M(x) \equiv \omega \in(-a, a)$ and the nonlinearity (the so-called Soler model)

$$
F(w)=\frac{1}{2} H(\tilde{w} w), H \in C^{2}(\mathbb{R}, \mathbb{R}), H(0)=0, \tilde{w} w:=(\beta w, w)_{\mathbb{C}^{4}},
$$

by using shooting methods. Such kind of nonlinearities were later studied in [17], where for the first time variational methods were applied to such problems (in fact, [17] also considered certain more general super-linear subcritical $F(w)$ independent of $x)$. If the equation is periodic, that is, $M(x)$ and $F(x, w)$ depend periodically on $x$, by using a critical point theory the paper [7] established also the existence and multiplicity of solutions of (1.2) with scalar potentials of the type $M(x)=V(x) \beta$. Concerning non-periodic potentials (typically, Coulomb-type potentials), [15] considered some asymptotically linear nonlinearities, and [16] treated superlinear and subcritical nonlinearities with mainly the limits of $M(x)$ and $F(x, w)$ existing as $|x| \rightarrow \infty$.

For small $\hbar$, the standing waves are referred to as semi-classical states. To describe the transition from quantum to classical mechanics, the existence of solutions $w_{\hbar}, \hbar$ small, possesses an important physical interest. Recently, the paper [14] studied the existence of a family of ground states of the problem

$$
-i \hbar \alpha \cdot \nabla w+a \beta w=W(x)|w|^{q-2} w, \quad(q \in(2,3))
$$

for all $\hbar$ small, and showed that the family concentrates around the maxima of $W(x)$ as $\hbar \rightarrow 0$.

To our knowledge, there are no results studying the existence and concentration phenomenon of semiclassical solutions for nonlinear Dirac equations involving the critical exponent of the relevant Sobolev embedding. Since the pioneering paper by Brezis-Nirenberg [8] on elliptic boundary value problems involving the critical Sobolev exponent, there have been a large number of works in this direction. In particular, many papers have been devoted to studying critical Schrödinger equations (not semiclassical case), see, e.g. [30] and the references therein.

The main objective of this paper is to study such situations for critical Dirac equations. For describing our study we present first some special consequences of the more general results of the paper. First, consider the problem

$$
-i \hbar \alpha \cdot \nabla u+a \beta u=W(x)\left(|u|^{q-2}+|u|\right) u .
$$


THEOREM 1.1. Let $q \in(2,3)$ and assume that $W$ satisfies

$\left(W_{0}\right) W \in C^{1}\left(\mathbb{R}^{3}, \mathbb{R}\right)$, inf $W>0$, and $\pi>\pi_{\infty}$.

Then there exists $\pi_{0}>0$ such that, if $\pi_{\infty}>\pi_{0}$, for sufficiently small $\hbar>0$, (1.4) possesses a least energy solutions $w_{\hbar} \in \bigcap_{s \geq 2} W^{1, s}$. If additionally $\nabla W$ is bounded, then $w_{\hbar}$ satisfies:

$\left(a_{1}\right)$ There exists a maximum point $x_{\hbar}$ of $\left|w_{\hbar}\right|$ with $\lim _{\hbar \rightarrow 0} \operatorname{dist}\left(x_{\hbar}, \mathscr{W}\right)=0$, such that, for some $c, C>0$

$$
\left|w_{\hbar}(x)\right| \leq C \exp \left(-\frac{c}{\hbar}\left|x-x_{\hbar}\right|\right) .
$$

$\left(a_{2}\right)$ Setting $v_{\hbar}(x):=w_{\hbar}\left(\hbar x+x_{\hbar}\right)$, for any sequence $\hbar \rightarrow 0, v_{\hbar}$ converges in $H^{1}$ to a least energy solution of

$$
-i \alpha \cdot \nabla v+a \beta v=\pi\left(|v|^{q-2}+|v|\right) v .
$$

In fact, one can choose

$$
\pi_{0}=a\left(\frac{6 \gamma_{q}}{S^{3 / 2}}\right)^{(q-2) / 2(3-q)}
$$

where here (and in the sequel) $S$ is the best Sobolev embedding constant:

$$
S|u|_{L^{6}}^{2} \leq|\nabla u|_{L^{2}}^{2} \quad \text { for } u \in H^{1}\left(\mathbb{R}^{3}\right),
$$

and $\gamma_{q}$ denotes the least energy of the ground state for the superlinear subcritical equation (which exists, see $[17,16]$ )

$$
-i \alpha \cdot \nabla u+\beta u=|u|^{q-2} u .
$$

We also consider the equation with linear potential

$$
-i \hbar \alpha \cdot \nabla u+a \beta u+V(x) u=|u|^{q-2} u+|u| u .
$$

THEOREM 1.2. Let $q \in(2,3)$ and assume that $V$ satisfies $\tau>-a$ and

$\left(V_{0}\right) V \in C^{1}\left(\mathbb{R}^{3}, \mathbb{R}\right), V(x) \leq 0$ and $\tau<\tau_{\infty}$.

Then there is $\tau_{0}>0$ such that, if $\left(a+\tau_{\infty}\right)^{12-5 q}<\tau_{0}$, for sufficiently small $\hbar>$ 0 , (1.7) possesses a least energy solutions $w_{\hbar} \in \bigcap_{s \geq 2} W^{1, s}$. If additionally $\nabla V$ is bounded, and either $12 \geq 5 q$ or $(a+\tau)^{12-5 q}<\tau_{0}$, then $w_{\hbar}$ satisfies:

$\left(a_{1}\right)$ There exists a maximum point $x_{\hbar}$ of $\left|w_{\hbar}\right|$ with $\lim _{\hbar \rightarrow 0} \operatorname{dist}\left(x_{\hbar}, \mathscr{V}\right)=0$, such that, for some $c, C>0,(1.5)$ holds.

$\left(a_{2}\right)$ Setting $v_{\hbar}(x):=w_{\hbar}\left(\hbar x+x_{\hbar}\right)$, for any sequence $\hbar \rightarrow 0, v_{\hbar}$ converges in $H^{1}$ to a least energy solution of

$$
-i \alpha \cdot \nabla v+a \beta v+\tau v=\left(|v|^{q-2}+|v|\right) v
$$
above.

In fact, one may take $\tau_{0}=a^{12-5 q} \pi_{0}^{-2(3-q)}$ where $\pi_{0}$ is the number defined

What will happen if $\tau=-a$ ?

TheOREM 1.3. Let $\left(V_{0}\right)$ be satisfied, $\tau=-a$ and $q \in(2,3)$. Then for sufficiently small $\hbar>0,(1.7)$ possesses a least energy solutions $w_{\hbar} \in \bigcap_{s \geq 2} W^{1, s}$. In addition, $w_{\hbar} \rightarrow 0$ in $H^{1}$ as $\hbar \rightarrow 0$. 
REMARK 1.4. The concentration phenomena showed in Theorems 1.1 and 1.2 can be re-described as follows: There is $\sigma>0$ such that, for any given $\delta, r>0$, there exists $\hbar_{0}>0$ satisfying

$$
\begin{aligned}
& \sup _{x \in N_{r}(\mathscr{A})}\left|w_{\hbar}(x)\right| \geq \sigma \\
& \sup _{x \in N_{r}^{c}(\mathscr{A})}\left|w_{\hbar}(x)\right| \leq \delta
\end{aligned}
$$

provided $0<\hbar \leq \hbar_{0}$, where $\mathscr{A}=\mathscr{V}$ in case $\left(V_{0}\right)$ and $\mathscr{A}=\mathscr{W}$ in case $\left(W_{0}\right), N_{r}(\mathscr{A})$ denotes a neighborhood of $\mathscr{A}$ with radius $r$, and $N_{r}^{c}(\mathscr{A})=\mathbb{R}^{3} \backslash N_{r}(\mathscr{A})$.

Our argument is variational: the semiclassical solutions are obtained as critical points of an energy functional $\digamma_{\hbar}$ associated to an equivalent problem of (1.1). Different from the Laplacian in the Schrödinger equation, the Dirac operator is unbounded from above and below. As a result, the functional $\digamma_{\hbar}$ is strongly indefinite and, hence, possesses an infinite-dimensional linking structure instead of a Mountain-Pass. Our arguments will be based on a suitable functional analytic framework. The linking structure yields a minimax value $c_{\hbar}$ for $\digamma_{\hbar}$. Since the problem is posed on the whole space $\mathbb{R}^{3}, \digamma_{\hbar}$ does not satisfy the general Palais-Smale condition, and so it cannot be directly concluded that $c_{\hbar}$ is a critical value. This will be checked via a reduced functional $I_{\hbar}$ with Nehari manifold $\mathscr{N}_{\hbar}$, which is such that $c_{\hbar}$ is nothing but the minimum of $I_{\hbar}$ on $\mathscr{N}_{\hbar}$. Comparing with [14], since the solutions depend not only on the linear potential but also on the nonlinear one, the present arguments are more delicate. One new ingredient is a comparison of $c_{\hbar}$ with the least energy of a class of limit problems. Another is an estimate for $c_{\hbar}$ through a discussion about some auxiliary functionals. And the third is a boundedness estimate for the maximum of the semiclassical ground states. Since either the linear part or the nonlinear one is not invariant under the $\mathbb{R}^{3}$-group action, such an estimate enables us to establish the concentration phenomena.

2. The main results. Now we describe more precisely the main results. Writing $\varepsilon=\hbar$, we are concerned with the equation

$$
-i \varepsilon \alpha \cdot \nabla w+a \beta w+V(x) w=W(x)(g(|w|)+|w|) w .
$$

On the nonlinear field, writing $G(|w|):=\int_{0}^{|w|} g(s) s d s$, we consider the following hypotheses:

$\left(g_{1}\right) g(0)=0, g \in C^{1}(0, \infty), g^{\prime}(s)>0$ for $s>0$, and there exist $p \in(2,3), c_{1}>0$ such that $g(s) \leq c_{1}\left(1+s^{p-2}\right)$ for $s \geq 0$

$\left(g_{2}\right)$ there exist $q>2, \theta>2$ and $c_{0}>0$ such that $c_{0} s^{q} \leq G(s) \leq \frac{1}{\theta} g(s) s^{2}$ for all $s>0$.

Clearly, the power function $g(s)=s^{q-2}$ for $s \geq 0$ satisfies these assumptions. Set

$$
\mathcal{R}_{q}:=\left(\frac{S^{3 / 2} c_{0}^{2 /(q-2)}}{6 \gamma_{q}}\right)^{q-2}
$$

where $q$ and $c_{0}$ are the constants from $\left(g_{2}\right)$, and $\gamma_{q}$ is the least energy of (1.6). On the linear fields we will use the following hypotheses:

$\left(P_{0}\right) V, W \in C^{1}\left(\mathbb{R}^{3}, \mathbb{R}\right), \quad V(x) \leq 0, \quad \inf W>0$

$\left(\hat{P}_{0}\right) \tau>-a$ and $\left(\frac{a+\tau_{\infty}}{a}\right)^{12-5 q}\left(\frac{a}{\pi_{\infty}}\right)^{2(3-q)}<\mathcal{R}_{q}$;

$\left(P_{1}\right) \pi>\pi_{\infty}$ and $\min _{x \in \mathscr{W}} V(x) \leq \tau_{\infty} ;$ or 
$\left(P_{2}\right) \tau<\tau_{\infty}$ and $\max _{x \in \mathscr{Y}} W(x) \geq \pi_{\infty}$

These conditions suffice for the existence of semiclassical solutions. In order to show the concentration phenomenon we require a further technical assumption:

$\left(P_{3}\right) \nabla V$ and $\nabla W$ are bounded, and

$$
\text { either } 5 q \leq 12, \text { or }\left(\frac{a+\tau}{a}\right)^{12-5 q}\left(\frac{a}{\pi}\right)^{2(3-q)}<\mathcal{R}_{q}
$$

TheOREM 2.1. Let $\left(g_{1}\right)-\left(g_{2}\right),\left(P_{0}\right)-\left(\hat{P}_{0}\right)$, and either $\left(P_{1}\right)$ or $\left(P_{2}\right)$ be satisfied. Then, for sufficiently small $\varepsilon>0,(2.1)$ possesses a least energy solution $w_{\varepsilon} \in$ $\bigcap_{s \geq 2} W^{1, s}$.

If additionally $\left(P_{3}\right)$ also holds and $\mathscr{V} \cap \mathscr{W} \neq \emptyset$, then $w_{\varepsilon}$ satisfies:

$\left(a_{1}\right)$ There exists a maximum point $x_{\varepsilon}$ of $\left|w_{\varepsilon}\right|$ with $\lim _{\varepsilon \rightarrow 0} \operatorname{dist}\left(x_{\varepsilon}, \mathscr{V} \cap \mathscr{W}\right)=0$, such that, for some $c, C>0$

$$
\left|w_{\varepsilon}(x)\right| \leq C \exp \left(-\frac{c}{\varepsilon}\left|x-x_{\varepsilon}\right|\right) .
$$

$\left(a_{2}\right)$ Setting $v_{\varepsilon}(x):=w_{\varepsilon}\left(\varepsilon x+x_{\varepsilon}\right)$, $v_{\varepsilon}$ converges in $H^{1}$ (up to subsequences) to a least energy solution of

$$
-i \alpha \cdot \nabla v+a \beta v+\tau v=\pi(g(|v|)+|v|) v .
$$

More generally, also if $\mathscr{V} \cap \mathscr{W} \neq \emptyset$ is not necessarily satisfied, we can describe certain concentration phenomena. To this end we introduce the following notations: in case $\left(P_{1}\right)$ set $\tau_{w}=\min _{x \in \mathscr{W}} V(x)$ and

$$
\mathscr{A}_{w}:=\left\{x \in \mathscr{W}: V(x)=\tau_{w}\right\} \cup\left\{x \notin \mathscr{W}: V(x)<\tau_{w}\right\}
$$

in case $\left(P_{2}\right)$ set $\pi_{v}=\max _{x \in \mathscr{V}} W(x)$ and

$$
\mathscr{A}_{v}:=\left\{x \in \mathscr{V}: W(x)=\pi_{v}\right\} \cup\left\{x \notin \mathscr{V}: W(x)>\pi_{v}\right\} .
$$

Obviously, $\mathscr{A}_{v}$ and $\mathscr{A}_{w}$ are bounded. Moreover, if $\mathscr{V} \cap \mathscr{W} \neq \emptyset$ then $\mathscr{A}_{v}=\mathscr{A}_{w}=\mathscr{V} \cap \mathscr{W}$.

First, consider the equation with the nonlinear potential $W$ leading the behavior.

TheOrem 2.2. Let $\left(g_{1}\right)-\left(g_{2}\right),\left(P_{0}\right)-\left(\hat{P}_{0}\right)$ and $\left(P_{1}\right)$ be satisfied. Then, for sufficiently small $\varepsilon>0$, (2.1) possesses a least energy solution $w_{\varepsilon} \in \bigcap_{s \geq 2} W^{1, s}$. If additionally $\left(P_{3}\right)$ also holds, then $w_{\varepsilon}$ satisfies:

$\left(a_{1}\right)$ There exists a maximum point $x_{\varepsilon}$ of $\left|w_{\varepsilon}\right|$ with $\lim _{\varepsilon \rightarrow 0} \operatorname{dist}\left(x_{\varepsilon}, \mathscr{A}_{w}\right)=0$, such that (2.2) holds. for some constants $c, C>0$.

$\left(a_{2}\right)$ Setting $v_{\varepsilon}(x):=w_{\varepsilon}\left(\varepsilon x+x_{\varepsilon}\right)$, for any sequence $x_{\varepsilon} \rightarrow x_{0}$ as $\varepsilon \rightarrow 0$, $v_{\varepsilon}$ converges in $H^{1}$ to a least energy solution of

$$
-i \alpha \cdot \nabla v+a \beta v+V\left(x_{0}\right) v=W\left(x_{0}\right)(g(|v|)+|v|) v .
$$

Next, consider the equation with the linear potential $V$ leading the behavior.

THEOREM 2.3. Let $\left(g_{1}\right)-\left(g_{2}\right),\left(P_{0}\right)-\left(\hat{P}_{0}\right)$ and $\left(P_{2}\right)$ be satisfied. Then for sufficiently small $\varepsilon>0$, (2.1) possesses a least energy solution $w_{\varepsilon} \in \bigcap_{s \geq 2} W^{1, s}$. If in addition also $\left(P_{3}\right)$ holds, then $w_{\varepsilon}$ satisfies

$\left(a_{1}\right)$ There exists a maximum point $x_{\varepsilon}$ of $\left|w_{\varepsilon}\right|$ with $\lim _{\varepsilon \rightarrow 0} \operatorname{dist}\left(x_{\varepsilon}, \mathscr{A}_{v}\right)=0$, such that, for some $c, C>0,(2.2)$ holds. 
$\left(a_{2}\right)$ Setting $v_{\varepsilon}(x):=w_{\varepsilon}\left(\varepsilon x+x_{\varepsilon}\right)$, for any sequence $x_{\varepsilon} \rightarrow x_{0}$ as $\varepsilon \rightarrow 0$, $v_{\varepsilon}$ converges in $H^{1}$ to a least energy solution of (2.4).

It is clear that Theorem 2.1 is a consequence of Theorems 2.2 and 2.3.

REMARK 2.4. We point out that if both $\left(P_{1}\right)$ and $\left(P_{2}\right)$ are satisfied, then in general it happens that (2.1) possesses two families of semiclassical ground states, one concentrating on $\mathscr{A}_{v}$ and another on $\mathscr{A}_{w}$.

Finally, we consider the case $\left(P_{2}\right)$ with $\tau=-a$.

TheOREm 2.5. Let $\left(g_{1}\right)-\left(g_{2}\right),\left(P_{0}\right)$ and $\left(P_{2}\right)$ be satisfied. Assume $\tau=-a$. Then, for sufficiently small $\varepsilon>0,(2.1)$ possesses a least energy solution $w_{\varepsilon} \in \bigcap_{s \geq 2} W^{1, s}$. Moreover, $w_{\varepsilon} \rightarrow 0$ in $H^{1}$ as $\varepsilon \rightarrow 0$.

Theorem 2.2 applies to the equation (2.1) with $\left(V_{0}\right)$ and $W(x)$ being constant, in particular, Theorem 1.1 is a corollary of Theorem 2.2. Likely, Theorem 2.3 applies to the equation (2.1) with $\left(W_{0}\right)$ and $V(x)$ being constant, so Theorem 1.2 is a particular case of Theorems 2.3. It is clear that Theorem 1.3 is a special case of Theorem 2.5.

REMARK 2.6. The formulas in $\left(\hat{P}_{0}\right)$ and $\left(P_{3}\right)$ are equivalent, respectively, to

$$
\begin{aligned}
& \pi_{\infty}>a\left(\mathcal{R}_{q}^{-1}\left(\frac{a+\tau_{\infty}}{a}\right)^{12-5 q}\right)^{1 / 2(3-q)}, \\
& \pi>a\left(\mathcal{R}_{q}^{-1}\left(\frac{a+\tau}{a}\right)^{12-5 q}\right)^{1 / 2(3-q)} .
\end{aligned}
$$

(The latter follows from the former if $12 \geq 5 q$; this is why we made the assumption only if $12<5 q$.)

Observe that, setting $u(x)=w(\varepsilon x), V_{\varepsilon}(x)=V(\varepsilon x)$ and $W_{\varepsilon}(x)=W(\varepsilon x)$, the equation (2.1) is equivalent to the following

$$
-i \alpha \cdot \nabla u+a \beta u+V_{\varepsilon}(x) u=W_{\varepsilon}(x)(g(|u|)+|u|) u
$$

We will in the sequel focus on these equivalent problems.

3. Variational setting. In what follows by $|\cdot|_{q}$ we denote the usual $L^{q}$-norm, and $(\cdot, \cdot)_{2}$ the usual $L^{2}$-inner product. Let $A_{a}=-i \alpha \cdot \nabla+a \beta$ denote the selfadjoint operator on $L^{2}\left(\mathbb{R}^{3}, \mathbb{C}^{4}\right)$ with domain $\mathcal{D}\left(A_{a}\right)=H^{1}\left(\mathbb{R}^{3}, \mathbb{C}^{4}\right)$. A Fourier analysis shows that $\sigma\left(A_{a}\right)=\sigma_{c}\left(A_{a}\right)=\mathbb{R} \backslash(-a, a)$ where $\sigma(\cdot)$ and $\sigma_{c}(\cdot)$ denote the spectrum and continuous spectrum. Thus the space $L^{2}$ possesses the orthogonal decomposition:

$$
L^{2}=L^{-} \oplus L^{+}, \quad u=u^{-}+u^{+}
$$

so that $A_{a}$ is negative definite (resp. positive definite) in $L^{-}$(resp. $L^{+}$). Let $E:=$ $\mathcal{D}\left(\left|A_{a}\right|^{1 / 2}\right)=H^{1 / 2}$ be equipped with the inner product

$$
(u, v)=\Re\left(\left|A_{a}\right|^{1 / 2} u,\left|A_{a}\right|^{1 / 2} v\right)_{2}
$$

and the induced norm $\|u\|=(u, u)^{1 / 2}$, where $\left|A_{a}\right|$ and $\left|A_{a}\right|^{1 / 2}$ denote respectively the absolute value of $A_{a}$ and the square root of $\left|A_{a}\right|$. Since $\sigma\left(A_{a}\right) \subset \mathbb{R} \backslash(-a, a)$, one has

$$
a|u|_{2}^{2} \leq\|u\|^{2} \quad \text { for all } u \in E .
$$

Note that this norm is equivalent to the usual $H^{1 / 2}$-norm, hence $E$ embeds continuously into $L^{q}$ for all $q \in[2,3]$ and compactly into $L_{l o c}^{q}$ for all $q \in[1,3)$. It is clear that $E$ possesses the following decomposition

$$
E=E^{-} \oplus E^{+} \quad \text { with } \quad E^{ \pm}=E \cap L^{ \pm},
$$


orthogonal with respect to both $(\cdot, \cdot)_{2}$ and $(\cdot, \cdot)$ inner products. This decomposition induces also a natural decomposition of $L^{q}$, hence there is $b_{q}>0$ such that

$$
b_{q}\left|u^{ \pm}\right|_{q}^{q} \leq|u|_{q}^{q} \text { for all } u \in E
$$

In the sequel, set $\mathbb{R}^{+}=[0, \infty)$, and define, for any $u \in E^{+} \backslash\{0\}$,

$$
E_{u}:=E^{-} \oplus \mathbb{R} u, \quad \hat{E}_{u}:=E^{-} \oplus \mathbb{R}^{+} u .
$$

Let $S$ and $S_{p}$ denote the following Sobolev embedding constants:

$$
\begin{aligned}
& S|u|_{6}^{2} \leq|\nabla u|_{2}^{2} \\
& S_{p}|u|_{p}^{2} \leq \int_{\mathbb{R}^{3}}|\nabla u|^{2}+|u|^{2} \quad, \quad \text { for all } u \in H^{1} .
\end{aligned}
$$

Lemma 3.1. Let $A_{\delta}:=-i \alpha \cdot \nabla+\delta \beta$ for all $\delta \neq 0$. Denoting $p_{t}=6 /(3-2 t)$ for $t \in[0,1]$, one has $|u|_{p_{t}} \leq\left.\left. S^{-t / 2}|| A_{\delta}\right|^{t} u\right|_{2}$.

Proof. See [7]. We consider two interpolation couples $\left\{Y_{0}, Y_{1}\right\}$ and $\left\{X_{0}, X_{1}\right\}$ with $Y_{0}=L^{2}, Y_{1}=L^{6}$, and $X_{0}=L^{2}=\mathcal{D}\left(\left|A_{\delta}\right|^{0}\right), X_{1}=\mathcal{D}\left(\left|A_{\delta}\right|\right)$. Let $Y_{+}=Y_{0}+Y_{1}$ be equipped with the norm

$$
\|u\|_{+}:=\inf \left\{|v|_{2}+|w|_{6}: u=v+w, v \in Y_{0}, w \in Y_{1}\right\}
$$

and $X_{+}=X_{0}+X_{1}$ be equipped with the norm

$$
\|u\|_{+}:=\inf \left\{|v|_{2}+\|w\|_{X_{1}}: u=v+w, v \in X_{0}, w \in X_{1}\right\} .
$$

Consider the (complex) interpolation spaces $Y_{t}=L^{p_{t}}$ and $X_{t}=\mathcal{D}\left(\left|A_{\delta}\right|^{t}\right)$. Let $\imath$ : $X_{+} \rightarrow Y_{+}$be the embedding operator. Then $\imath\left(X_{1}\right) \subset Y_{1}$ and the Sobolev embedding theorem yields $\|\imath\|_{\mathcal{L}\left(X_{1}, Y_{1}\right)} \leq S^{-1 / 2}$. The Calderón-Lions interpolation theorem ([26]) now implies $\imath\left(X_{t}\right) \subset Y_{t}$ and

$$
\|\imath\|_{\mathcal{L}\left(X_{t}, Y_{t}\right)} \leq\|\imath\|_{\mathcal{L}\left(X_{0}, Y_{0}\right)}^{1-t}\|\imath\|_{\mathcal{L}\left(X_{1}, Y_{1}\right)}^{t}=\|\imath\|_{\mathcal{L}\left(X_{1}, Y_{1}\right)}^{t}=S^{-t / 2}
$$

ending the proof

REMARK 3.2. 1) We note in particular that $S^{1 / 2}|u|_{3}^{2} \leq \|\left.\left.(-i \alpha \cdot \nabla+\delta \beta)\right|^{1 / 2} u\right|_{2} ^{2}$ for any $\delta$ and all $u \in E$.

2) One verifies similarly that, for any $p \in[2,3)$,

$$
\begin{gathered}
\delta^{(6-2 p) / p} S_{2 p /(4-p)}^{1 / 2}|u|_{p}^{2} \leq\left\|\left.A_{\delta}\right|^{1 / 2} u\right\|_{2}^{2} . \\
S_{2 p /(4-p)}^{1 / 2}|u|_{p}^{2} \leq\left\|\left.A_{1}\right|^{1 / 2} u\right\|_{2}^{2} .
\end{gathered}
$$

In virtue of the assumptions $\left(g_{1}\right)-\left(g_{2}\right)$, for any $\delta>0$ with $\delta<(a-\tau) / 4$, there exist $r_{\delta}>0, c_{\delta}>0$ and $c_{\delta}^{\prime}>0$ such that

$$
\left\{\begin{array}{l}
g(s)<\delta \quad \text { for all } 0 \leq s \leq r_{\delta} \\
G(|u|) \geq c_{\delta}|u|^{\theta}-\delta|u|^{2} \quad \text { for all } u \in \mathbb{C}^{4} \\
G(|u|) \leq \delta|u|^{2}+c_{\delta}^{\prime}|u|^{p} \quad \text { for all } u \in \mathbb{C}^{4}
\end{array}\right.
$$


Denote $f(s):=g(s)+s$ for $s \geq 0$, and

$$
F(|u|):=\int_{0}^{|u|} f(s) s d s=G(|u|)+\frac{1}{3}|u|^{3} .
$$

On $E$ we set $a(u, v):=\left(\left|A_{a}\right|^{1 / 2}\left(u^{+}-u^{-}\right),\left|A_{a}\right|^{1 / 2} v\right)_{2}=\left(u^{+}-u^{-}, v\right)$, and $a(u)=$ $a(u, u)=\left\|u^{+}\right\|^{2}-\left\|u^{-}\right\|^{2}$. In the sequel, for convenience, we will write also $\int_{\mathbb{R}^{3}}\left\langle A_{a} u, v\right\rangle$ for $a(u, v)$. Define the functional

$$
\begin{aligned}
\Phi_{\varepsilon}(u) & =\frac{1}{2} a(u)+\frac{1}{2} \int_{\mathbb{R}^{3}} V_{\varepsilon}(x)|u|^{2}-\int_{\mathbb{R}^{3}} W_{\varepsilon}(x) F(|u|) \\
& =\frac{1}{2}\left(\left\|u^{+}\right\|^{2}-\left\|u^{-}\right\|^{2}\right)+\frac{1}{2} \int_{\mathbb{R}^{3}} V_{\varepsilon}(x)|u|^{2}-\int_{\mathbb{R}^{3}} W_{\varepsilon}(x) F(|u|) .
\end{aligned}
$$

Plainly, $\Phi_{\varepsilon} \in C^{2}(E, \mathbb{R})$.

LEMMA 3.3. Critical points of $\Phi_{\varepsilon}$ are solutions of (2.5).

Proof. Observe that, for any $u, v \in E$,

$$
\begin{aligned}
& \left.\frac{d}{d s} \Phi_{\varepsilon}(u+s v)\right|_{s=0} \\
= & \Re a(u, v)+\Re \int_{\mathbb{R}^{3}} V_{\varepsilon}(x)\langle u, v\rangle-\Re \int_{\mathbb{R}^{3}} W_{\varepsilon}(x) f(|u|)\langle u, v\rangle \\
= & \left(u^{+}-u^{-}, v\right)+\Re \int_{\mathbb{R}^{3}}\left(V_{\varepsilon}(x)-W_{\varepsilon}(x) f(|u|)\right)\langle u, v\rangle .
\end{aligned}
$$

Let $u \in E$ be a critical point of $\Phi_{\varepsilon}$. For any real vector $v \in C_{0}^{\infty}\left(\mathbb{R}^{3}, \mathbb{R}^{4}\right)$ one has formally

$$
\begin{aligned}
0 & =\left(u^{+}-u^{-}, v\right)+\Re \int_{\mathbb{R}^{3}}\left(V_{\varepsilon}(x)-W_{\varepsilon}(x) f(|u|)\right)\langle u, v\rangle \\
& =\left(\Re\left(A_{a} u\right)+V_{\varepsilon}(\Re u)-W_{\varepsilon} f(|u|)(\Re u), v\right)_{2}
\end{aligned}
$$

and

$$
\begin{aligned}
0 & =\left(u^{+}-u^{-}, i v\right)+\Re \int_{\mathbb{R}^{3}}\left(V_{\varepsilon}(x)-W_{\varepsilon}(x) f(|u|)\right)\langle u, i v\rangle \\
& =\left(\Im\left(A_{a} u\right)+V_{\varepsilon}(\Im u)-W_{\varepsilon} f(|u|)(\Im u), v\right)_{2} .
\end{aligned}
$$

Hence, for any general $v \in C_{0}^{\infty}\left(\mathbb{R}^{3}, \mathbb{C}^{4}\right)$, there holds

$$
0=\left(A_{a} u+V_{\varepsilon} u-W_{\varepsilon} f(|u|) u, v\right)_{2}
$$

which implies that $u$ is a weak solution of (2.5). Now a standard regularity argument shows that $u$ is in fact a solution of (2.5).

Note that the functional $\Psi_{\varepsilon}(u):=\int_{\mathbb{R}^{3}} W_{\varepsilon}(x) F(|u|)$ is weakly sequentially lower semi-continuous, and $\Phi_{\varepsilon}^{\prime}$ is weakly sequentially continuous.

It is easy to check by using $(3.1)-(3.3)$ that the functional $\Phi_{\varepsilon}$ possesses the linking structure:

LEMMA 3.4. $\Phi_{\varepsilon}$ possesses the linking structure:

1) There exist $r>0$ and $\rho>0$ both independent of $\varepsilon$ such that $\left.\Phi_{\varepsilon}\right|_{B_{r}^{+}}(u) \geq 0$ and $\left.\Phi_{\varepsilon}\right|_{S_{r}^{+}} \geq \rho$, where $B_{r}^{+}=\left\{u \in E^{+}:\|u\| \leq r\right\}$ and $S_{r}^{+}=\left\{u \in E^{+}:\|u\|=r\right\}$; 
2) For any $e \in E^{+} \backslash\{0\}$, there exist $R_{e}>0$ and $C=C_{e}>0$ both independent of $\varepsilon$ such that $\Phi_{\varepsilon}(u)<0$ for all $u \in \hat{E}_{e} \backslash B_{R}$ and $\max \Phi_{\varepsilon}\left(\hat{E}_{e}\right) \leq C$.

Define the following minimax value (see $[23,28,6]$ )

$$
c_{\varepsilon}:=\inf _{e \in E^{+} \backslash\{0\}} \max _{u \in E_{e}} \Phi_{\varepsilon}(u)=\inf _{e \in E^{+} \backslash\{0\}} \max _{u \in \hat{E}_{e}} \Phi_{\varepsilon}(u) .
$$

As a consequence of Lemma 3.4 we have

Lemma 3.5. There is $C>0$ independent of $\varepsilon$ such that $\rho \leq c_{\varepsilon}<C$.

Proof. By 1) of Lemma 3.4 and the definition of $c_{\varepsilon}$ one has $c_{\varepsilon} \geq \rho$. Take $e \in E^{+}$ with $\|e\|=1$. It follows from 2) of Lemma 3.4 the following

$$
c_{\varepsilon} \leq C \equiv C_{e},
$$

ending the proof.

Recall that a sequence $\left\{u_{n}\right\} \subset E$ is said to be a $(P S)_{c}, c \in \mathbb{R}$, sequence for $\Phi_{\varepsilon}$ if $\Phi_{\varepsilon}\left(u_{n}\right) \rightarrow c$ and $\Phi_{\varepsilon}^{\prime}\left(u_{n}\right) \rightarrow 0$, and $\Phi_{\varepsilon}$ is said to satisfy the $(P S)_{c}$ condition if any $(P S)_{c}$ sequence for $\Phi_{\varepsilon}$ has a convergent subsequence. With Lemma 3.4 and by a linking argument it follows that $\Phi_{\varepsilon}$ has a $(P S)_{c_{\varepsilon}}$ sequence (see e.g. [13, 28]). Obviously, if $\Phi_{\varepsilon}$ satisfies the $(P S)_{c}$ condition then $c_{\varepsilon}$ is a critical value. Unfortunately, since there is no compact embedding from $H^{1 / 2}\left(\mathbb{R}^{3}\right)$ into $L^{p}\left(\mathbb{R}^{3}\right)$, the $(P S)$ condition does not in general hold, and we have to go through a more delicate analysis.

Motivated by Ackermann [1] (see also [16, 23, 28]), we consider, for a fixed $u \in E^{+}$, the map $\phi_{u}: E^{-} \rightarrow \mathbb{R}$ defined by

$$
\phi_{u}(v)=\Phi_{\varepsilon}(u+v) .
$$

Observe that, for any $v, w \in E^{-}$(recalling that $\left.-\tau=|V|_{\infty}\right)$,

$$
\begin{aligned}
\phi_{u}^{\prime \prime}(v)[w, w] & =-\|w\|^{2}+\int_{\mathbb{R}^{3}} V_{\varepsilon}(x)|w|^{2}-\Psi_{\varepsilon}^{\prime \prime}(u+v)[w, w] \\
& \leq-\frac{a+\tau}{a}\|w\|^{2}-\Psi_{\varepsilon}^{\prime \prime}(u+v)[w, w],
\end{aligned}
$$

and in addition

$$
\phi_{u}(v) \leq \frac{a-\tau}{2 a}\|u\|^{2}-\frac{a+\tau}{2 a}\|v\|^{2} .
$$

Therefore, there is a unique $h_{\varepsilon}(u) \in E^{-}$such that

$$
\phi_{u}\left(h_{\varepsilon}(u)\right)=\max _{v \in E^{-}} \phi_{u}(v) .
$$

It is clear that

$$
\begin{aligned}
0 & =\phi_{u}^{\prime}\left(h_{\varepsilon}(u)\right) v \\
& =-\left(h_{\varepsilon}(u), v\right)+\Re \int_{\mathbb{R}^{3}} V_{\varepsilon}(x)\left\langle u+h_{\varepsilon}(u), v\right\rangle-\Psi_{\varepsilon}^{\prime}\left(u+h_{\varepsilon}(u)\right) v
\end{aligned}
$$

for all $v \in E^{-}$, and

$$
v \neq h_{\varepsilon}(u) \quad \Leftrightarrow \quad \Phi_{\varepsilon}(u+v)<\Phi_{\varepsilon}\left(u+h_{\varepsilon}(u)\right) .
$$


For any $u \in E^{+}$and $v \in E^{-}$, setting $z=v-h_{\varepsilon}(u)$ and $\ell(t)=\phi_{u}\left(h_{\varepsilon}(u)+t z\right)$, one has $\ell(1)=\phi_{u}(v), \ell(0)=\phi_{u}\left(h_{\varepsilon}(u)\right.$ and $\ell^{\prime}(0)=0$. Thus $\ell(1)-\ell(0)=\int_{0}^{1}(1-t) \ell^{\prime \prime}(t) d t$. This implies that

$$
\begin{aligned}
& \phi_{u}(v)-\phi_{u}\left(h_{\varepsilon}(u)\right) \\
= & \left.\int_{0}^{1}(1-t) \phi_{u}^{\prime \prime}\left(h_{\varepsilon}(u)+t z\right)\right)[z, z] d t \\
= & -\int_{0}^{1}(1-t)\left(\|z\|^{2}-\int_{\mathbb{R}^{3}} V_{\varepsilon}(x)|z|^{2}+\int_{\mathbb{R}^{3}} W_{\varepsilon}(x) f\left(\left|u+h_{\varepsilon}(u)+t z\right|\right)|z|^{2}\right. \\
& \left.\quad+\int_{\mathbb{R}^{3}} W_{\varepsilon}(x) \frac{f^{\prime}\left(\left|u+h_{\varepsilon}(u)+t z\right|\right)}{\left|u+h_{\varepsilon}(u)+t z\right|}\left(\Re\left\langle u+h_{\varepsilon}(u)+t z, z\right\rangle\right)^{2}\right) d t
\end{aligned}
$$

hence,

$$
\begin{aligned}
& \Phi_{\varepsilon}\left(u+h_{\varepsilon}(u)\right)-\Phi_{\varepsilon}(u+v) \\
= & \frac{1}{2}\left(\|z\|^{2}-\int_{\mathbb{R}^{3}} V_{\varepsilon}(x)|z|^{2}\right) \\
& +\int_{0}^{1} \int_{\mathbb{R}^{3}}(1-t) W_{\varepsilon}(x)\left(f\left(\left|u+h_{\varepsilon}(u)+t z\right|\right)|z|^{2}\right. \\
& \left.+\frac{f^{\prime}\left(\left|u+h_{\varepsilon}(u)+t z\right|\right)}{\left|u+h_{\varepsilon}(u)+t z\right|}\left(\Re\left\langle u+h_{\varepsilon}(u)+t z, z\right\rangle\right)^{2}\right) .
\end{aligned}
$$

Define $I_{\varepsilon}: E^{+} \rightarrow \mathbb{R}$ by

$$
\begin{aligned}
I_{\varepsilon}(u) & =\Phi_{\varepsilon}\left(u+h_{\varepsilon}(u)\right) \\
& =\frac{1}{2}\left(\|u\|^{2}-\left\|h_{\varepsilon}(u)\right\|^{2}\right)+\frac{1}{2} \int_{\mathbb{R}^{3}} V_{\varepsilon}(x)\left|u+h_{\varepsilon}(u)\right|^{2}-\Psi_{\varepsilon}\left(u+h_{\varepsilon}(u)\right) .
\end{aligned}
$$

Set

$$
\mathscr{N}_{\varepsilon}:=\left\{u \in E^{+} \backslash\{0\}: I_{\varepsilon}^{\prime}(u) u=0\right\}
$$

LEMMA 3.6. For any $u \in E^{+} \backslash\{0\}$, there is a unique $t=t(u)>0$ such that $t(u) u \in \mathscr{N}_{\varepsilon}$.

Proof. See $[1,14,16]$.

LEMma 3.7. We have

$$
c_{\varepsilon}=\inf _{u \in \mathscr{N}_{\varepsilon}} I_{\varepsilon}(u)
$$

Proof. Indeed, given $e \in E^{+}$, if $u=v+s e \in E_{e}$ with $\Phi_{\varepsilon}(u)=\max _{z \in E_{e}} \Phi_{\varepsilon}(z)$ then the restriction $\left.\Phi_{\varepsilon}\right|_{E_{e}}$ of $\Phi_{\varepsilon}$ on $E_{e}$ satisfies $\left(\left.\Phi_{\varepsilon}\right|_{E_{e}}\right)^{\prime}(u)=0$ which implies $v=h_{\varepsilon}(s e)$ and $I_{\varepsilon}^{\prime}(s e)(s e)=\Phi_{\varepsilon}^{\prime}(u)(s e)=0$, i.e. se $\in \mathscr{N}_{\varepsilon}$. Thus $\inf I_{\varepsilon}\left(\mathscr{N}_{\varepsilon}\right) \leq c_{\varepsilon}$. On the other hand, if $w \in \mathscr{N}_{\varepsilon}$ then $\left(\left.\Phi_{\varepsilon}\right|_{E_{w}}\right)^{\prime}\left(w+h_{\varepsilon}(w)\right)=0$ so $c_{\varepsilon} \leq \max _{u \in E_{w}} \Phi_{\varepsilon}(u)=I_{\varepsilon}(w)$. Thus $\inf I_{\varepsilon}\left(\mathscr{N}_{\varepsilon}\right) \geq c_{\varepsilon}$. This proves the desired conclusion.

LemMA 3.8. For any $e \in E^{+} \backslash\{0\}$, there is $T_{e}>0$ independent of $\varepsilon>0$ such that $t_{\varepsilon} \leq T_{e}$ for $t_{\varepsilon}>0$ satisfying $t_{\varepsilon} e \in \mathscr{N}_{\varepsilon}$.

Proof. Since $I_{\varepsilon}^{\prime}\left(t_{\varepsilon} e\right)\left(t_{\varepsilon} e\right)=0$ one sees that the restriction of $\Phi_{\varepsilon}$ satisfies $\left(\left.\Phi_{\varepsilon}\right|_{E_{e}}\right)^{\prime}\left(t_{\varepsilon} e+\right.$ $\left.h_{\varepsilon}\left(t_{\varepsilon} e\right)\right)=0$. Thus

$$
\Phi_{\varepsilon}\left(t_{\varepsilon} e+h_{\varepsilon}\left(t_{\varepsilon} e\right)\right)=\max _{w \in E_{e}} \Phi_{\varepsilon}(w)
$$


This, together with Lemma 3.7 and 2) of Lemma 3.4, implies the desired conclusion.

Let $\mathscr{K}_{\varepsilon}:=\left\{u \in E: \Phi_{\varepsilon}^{\prime}(u)=0\right\}$ be the critical set of $\Phi_{\varepsilon}$. It is easy to see that if $K_{\varepsilon} \backslash\{0\} \neq \emptyset$ then

$$
c_{\varepsilon}=\inf \left\{\Phi_{\varepsilon}(u): u \in \mathscr{K}_{\varepsilon} \backslash\{0\}\right\}
$$

(see an argument of [16]). Using the same iterative argument of [17, Proposition 3.2] one obtains easily the following

Lemma 3.9. If $u \in \mathscr{K}_{\varepsilon}$ with $\left|\Phi_{\varepsilon}(u)\right| \leq C_{1}$ and $|u|_{2} \leq C_{2}$, then, for any $s \in[2, \infty)$, $u \in W^{1, s}\left(\mathbb{R}^{3}\right)$ with $\|u\|_{W^{1, s}} \leq \Lambda_{s}$ where $\Lambda_{s}$ depends only on $C_{1}, C_{2}$ and $s$.

Let $\mathscr{S}_{\varepsilon}$ be the set of all least energy solutions of $\Phi_{\varepsilon}$. If $u \in \mathscr{S}_{\varepsilon}$ then $\Phi_{\varepsilon}(u)=c_{\varepsilon}$ and a standard argument shows that $\mathscr{S}_{\varepsilon}$ is bounded in $E$, hence, $|u|_{2} \leq C_{2}$ for $u \in \mathscr{S}_{\varepsilon}$, some $C_{2}>0$ independent of $\varepsilon$. Therefore, as a consequence of Lemmas 3.7 and 3.9 we see that, for each $s \in[2, \infty)$, there is $C_{s}>0$ independent of $\varepsilon$ such that

$$
\|u\|_{W^{1, s}} \leq C_{s} \text { for all } u \in \mathscr{S}_{\varepsilon} \text {. }
$$

This, together with the Sobolev embedding theorem, implies that there is $C_{\infty}>0$ independent of $\varepsilon$ with

$$
|u|_{\infty} \leq C_{\infty} \text { for all } u \in \mathscr{S}_{\varepsilon} .
$$

4. Preliminary results. For proving our main theorems, we need some results on related autonomous equations. Recall that $G(|u|) \geq c_{0}|u|^{q}, q \in(2,3)$. For any $\mu \in(-a, a)$ and $\nu>0$, consider the equation

$$
-i \alpha \cdot \nabla u+a \beta u+\mu u=\nu(g(|u|)+|u|) u, \quad u \in H^{1}\left(\mathbb{R}^{3}, \mathbb{C}^{4}\right) .
$$

Its solutions are critical points of the functional

$$
\Gamma_{\mu \nu}^{*}(u):=\frac{1}{2} \int_{\mathbb{R}^{3}}\langle(-i \alpha \cdot \nabla+a \beta+\mu) u, u\rangle-\nu \int_{\mathbb{R}^{3}}\left(G(|u|)+\frac{1}{3}|u|^{3}\right)
$$

defined for $u \in E$. Let $\gamma_{\mu \nu}^{*}$ denote the linking level of $\Gamma_{\mu \nu}^{*}$.

Proposition 4.1. $\gamma_{\mu \nu}^{*}$ is attained provided $\mu \leq 0$ and

$$
\left(\frac{a+\mu}{a}\right)^{12-5 q}\left(\frac{a}{\nu}\right)^{2(3-q)}<\mathcal{R}_{q} .
$$

In order to prove this proposition we require a series of discussions.

4.1. The subcritical equation. Consider

$$
-i \alpha \cdot \nabla u+a \beta u+\mu u=\nu g(|u|) u, \quad u \in H^{1}\left(\mathbb{R}^{3}, \mathbb{C}^{4}\right) .
$$

The solutions are critical points of the functional

$$
\Gamma_{\mu \nu}(u):=\frac{1}{2}\left(\left\|u^{+}\right\|^{2}-\left\|u^{-}\right\|^{2}\right)+\frac{\mu}{2} \int_{\mathbb{R}^{3}}|u|^{2}-\nu \int_{\mathbb{R}^{3}} G(|u|)
$$

defined for $u=u^{-}+u^{+} \in E=E^{-} \oplus E^{+}$. Denote the critical set, the least energy, and the set of least energy solutions of $\Gamma_{\mu \nu}$ as follows

$$
\begin{aligned}
& \mathscr{L}_{\mu \nu}:=\left\{u \in E: \Gamma_{\mu \nu}^{\prime}(u)=0\right\} \\
& \gamma_{\mu \nu}:=\inf \left\{\Gamma_{\mu \nu}(u): u \in \mathscr{L}_{\mu \nu} \backslash\{0\}\right\}, \\
& \mathscr{R}_{\mu \nu}:=\left\{u \in \mathscr{L}_{\mu \nu}: \Gamma_{\mu \nu}(u)=\gamma_{\mu \nu},|u(0)|=|u|_{\infty}\right\} .
\end{aligned}
$$


The following lemma is from [16].

LEMMA 4.2. There hold the following

i) $\mathscr{L}_{\mu \nu} \neq \emptyset, \gamma_{\mu \nu}>0$, and $\mathscr{L}_{\mu \nu} \subset \bigcap_{s \geq 2} W^{1, s}$;

ii) $\gamma_{\mu \nu}$ is attained, and $\mathscr{R}_{\mu \nu}$ is compact in $H^{1}\left(\mathbb{R}^{3}, \mathbb{C}^{4}\right)$;

iii) there exist $C, c>0$ such that

$$
|u(x)| \leq C \exp (-c|x|) \quad \text { for all } x \in \mathbb{R}^{3}, u \in \mathscr{R}_{\mu \nu} .
$$

As before we introduce the following notations:

$$
\begin{array}{ll}
\mathscr{J}_{\mu \nu}: E^{+} \rightarrow E^{-}, & \Gamma_{\mu \nu}\left(u+\mathscr{J}_{\mu \nu}(u)\right)=\max _{v \in E^{-}} \Gamma_{\mu \nu}(u+v) ; \\
J_{\mu \nu}: E^{+} \rightarrow \mathbb{R}, \quad J_{\mu \nu}(u)=\Gamma_{\mu \nu}\left(u+\mathscr{J}_{\mu \nu}(u)\right) ; \\
\mathscr{M}_{\mu \nu}:=\left\{u \in E^{+} \backslash\{0\}: J_{\mu \nu}^{\prime}(u) u=0\right\} .
\end{array}
$$

Plainly, critical points of $J_{\mu \nu}$ and $\Gamma_{\mu \nu}$ are in one to one correspondence via the injective map $u \rightarrow u+\mathscr{J}_{\mu \nu}(u)$ from $E^{+}$into $E$. Clearly, $J_{\mu \nu}$ has the Mountain-pass structure.

Notice that, similar to (3.4), for $u \in E^{+}, v \in E^{-}$and $z=v-\mathscr{J}_{\mu \nu}(u)$, there holds

$$
\begin{aligned}
& \Gamma_{\mu \nu}\left(u+\mathscr{J}_{\mu \nu}(u)\right)-\Gamma_{\mu \nu}(u+v) \\
& =\frac{1}{2}\left(\|z\|^{2}-\mu|z|_{2}^{2}\right)+\nu \int_{0}^{1} \int_{\mathbb{R}^{3}}(1-t)\left(g\left(\left|u+\mathscr{J}_{\mu \nu}(u)+t z\right|\right)|z|^{2}\right. \\
& \left.\quad+\frac{g^{\prime}\left(\left|u+\mathscr{J}_{\mu \nu}(u)+t z\right|\right)}{\left|u+\mathscr{J}_{\mu \nu}(u)+t z\right|}\left(\Re\left\langle u+\mathscr{J}_{\mu \nu}(u)+t z, z\right\rangle\right)^{2}\right) .
\end{aligned}
$$

It is not difficult to check that, for each $u \in E^{+} \backslash\{0\}$, there is a unique $t=t(u)>0$ such that $t u \in \mathscr{M}_{\mu \nu}$ (see Lemma 3.6, or $[1,16]$ ).

LEMMA 4.3. Let $u \in \mathscr{M}_{\mu \nu}$ be such that $J_{\mu \nu}(u)=\gamma_{\mu \nu}$. Then

$$
\max _{w \in E_{u}} \Gamma_{\mu \nu}(w)=J_{\mu \nu}(u)
$$

Proof. Clearly, since $u+\mathscr{J}_{\mu \nu}(u) \in E_{u}$,

$$
J_{\mu \nu}(u)=\Gamma_{\mu \nu}\left(u+\mathscr{J}_{\mu \nu}(u)\right) \leq \max _{w \in E_{u}} \Gamma_{\mu \nu}(w) .
$$

On the other hand, for any $w=v+s u \in E_{u}$,

$$
\begin{aligned}
\Gamma_{\mu \nu}(w) & =\frac{1}{2}\|s u\|^{2}-\frac{1}{2}\|v\|^{2}+\frac{\mu}{2}|v+s u|_{2}^{2}-\nu \int_{\mathbb{R}^{3}} G(|v+s u|) \\
& \leq \Gamma_{\mu \nu}\left(s u+\mathscr{J}_{\mu \nu}(s u)\right)=J_{\mu \nu}(s u) .
\end{aligned}
$$

Thus, since $u \in \mathscr{M}_{\mu \nu}$

$$
\max _{w \in E_{u}} \Gamma_{\mu \nu}(w) \leq \max _{s \geq 0} J_{\mu \nu}(s u)=J_{\mu \nu}(u)
$$

giving the conclusion.

LEMmA 4.4. Let $\mu_{j} \in(-a, a)$ and $\nu_{j}>0, j=1,2$, with $\min \left\{\mu_{2}-\mu_{1}, \nu_{1}-\nu_{2}\right\} \geq 0$. Then $\gamma_{\mu_{1} \nu_{1}} \leq \gamma_{\mu_{2} \nu_{2}}$. If additionally $\max \left\{\mu_{2}-\mu_{1}, \nu_{1}-\nu_{2}\right\}>0$ then $\gamma_{\mu_{1} \nu_{1}}<\gamma_{\mu_{2} \nu_{2}}$. In particular, $\gamma_{\mu_{1} \nu_{j}}<\gamma_{\mu_{2} \nu_{j}}$ if $\mu_{1}<\mu_{2}$, and $\gamma_{\mu_{j} \nu_{1}}>\gamma_{\mu_{j} \nu_{2}}$ if $\nu_{1}<\nu_{2}$. 
Proof. Let $u \in \mathscr{L}_{\mu_{2} \nu_{2}}$ with $\Gamma_{\mu_{2} \nu_{2}}(u)=\gamma_{\mu_{2} \nu_{2}}$ and set $e=u^{+}$. Then

$$
\gamma_{\mu_{2} \nu_{2}}=\Gamma_{\mu_{2} \nu_{2}}(u)=\max _{w \in E_{e}} \Gamma_{\mu_{2} \nu_{2}}(w) .
$$

Let $u_{0} \in E_{e}$ be such that $\Gamma_{\mu_{1} \nu_{1}}\left(u_{0}\right)=\max _{w \in E_{e}} \Gamma_{\mu_{1} \nu_{1}}(w)$. One has

$$
\begin{aligned}
\gamma_{\mu_{2} \nu_{2}} & =\Gamma_{\mu_{2} \nu_{2}}(u) \geq \Gamma_{\mu_{2} \nu_{2}}\left(u_{0}\right) \\
& =\Gamma_{\mu_{1} \nu_{1}}\left(u_{0}\right)+\frac{1}{2}\left(\mu_{2}-\mu_{1}\right)\left|u_{0}\right|_{2}^{2}+\left(\nu_{1}-\nu_{2}\right) \int_{\mathbb{R}^{3}} G\left(\left|u_{0}\right|\right) \\
& \geq \gamma_{\mu_{1} \nu_{1}}+\frac{1}{2}\left(\mu_{2}-\mu_{1}\right)\left|u_{0}\right|_{2}^{2}+\left(\nu_{1}-\nu_{2}\right) \int_{\mathbb{R}^{3}} G\left(\left|u_{0}\right|\right)
\end{aligned}
$$

as claimed.

For later use, define, for $q \in(2,3)$,

$$
T_{q}:=\inf _{u \in E^{+} \backslash\{0\}} \max _{v \in E^{-}} \frac{a_{1}(u+v)}{|u+v|_{q}^{2}}
$$

where $a_{1}(z)=\int_{\mathbb{R}^{3}}\left\langle A_{1} z, z\right\rangle$, and consider the equation

$$
-i \alpha \cdot \nabla u+\beta u=|u|^{q-2} u
$$

with the least energy functional defined by

$$
\Gamma_{q}(u):=\frac{1}{2} a_{1}(u)-\frac{1}{q}|u|_{q}^{q}
$$

and the least energy denoted by $\gamma_{q}$. Set as before the induced map $\mathscr{J}_{q}: E^{+} \rightarrow E^{-}$, the functional $J_{q} \in C^{2}\left(E^{+}, \mathbb{R}\right): J_{q}(u)=\Gamma_{q}\left(u+\mathscr{J}_{q}(u)\right)$, and the manifold $\mathscr{M}_{q}$.

Lemma 4.5. For any $q \in(2,3), T_{q}$ is achieved at some $u$ which is a least energy solution of the equation (4.5). Moreover,

$$
T_{q}=\left(\frac{2 q \gamma_{q}}{q-2}\right)^{(q-2) / q}
$$

Proof. Set, for any $u \in E^{+}$,

$$
\psi_{u}(v)=\frac{a_{1}(u+v)}{|u+v|_{q}^{2}} \quad \text { and } \quad T_{q}(u)=\max _{v \in E^{-}} \psi_{u}(v)
$$

If $w \in E^{-}$with $\psi_{u}(w)=T_{q}(u)$, then, for $v \in E^{-}$,

$$
\begin{aligned}
0= & \psi_{u}^{\prime}(w) v \\
= & \frac{2}{|u+w|_{q}^{4}} \Re\left[a_{1}(u+w, v)|u+w|_{q}^{2}\right. \\
& \left.-a_{1}(u+w)|u+w|_{q}^{2-q} \int_{\mathbb{R}^{3}}|u+w|^{q-2}(u+w) \bar{v}\right]
\end{aligned}
$$


and

$$
\begin{aligned}
\psi_{u}^{\prime \prime}(w)[v, v]= & \frac{2}{|u+w|_{q}^{4}} \Re\left[a_{1}(v)|u+w|_{q}^{2}\right. \\
& -(q-2) a_{1}(u+w)|u+w|_{q}^{2-q} \int_{\mathbb{R}^{3}}|u+w|^{q-4}((u+w) \bar{v})^{2} \\
& +(q-2) a_{1}(u+w)|u+w|^{2-2 q}\left(\int_{\mathbb{R}^{3}}|u+w|^{q-2}(u+w) \bar{v}\right)^{2} \\
& \left.-a_{1}(u+w)|u+w|^{2-q} \int_{\mathbb{R}^{3}}|u+w|^{q-2}|v|^{2}\right] \\
\leq & \frac{2}{|u+w|_{q}^{4}} \Re\left[a_{1}(v)|u+w|_{q}^{2}\right. \\
& \left.-a_{1}(u+w)|u+w|^{2-q} \int_{\mathbb{R}^{3}}|u+w|^{q-2}|v|^{2}\right] .
\end{aligned}
$$

Here we have used the estimate (by Hölder inequality)

$$
\left(\int_{\mathbb{R}^{3}}|u+w|^{q-2}(u+w) \bar{v}\right)^{2} \leq|u+w|_{q}^{q} \int_{\mathbb{R}^{3}}|u+w|^{q-4}((u+w) \bar{v})^{2} .
$$

and $a_{1}(u+w)>0\left(\right.$ since $\left.T_{q}(u)>0\right)$. Therefore, $\psi_{u}$ attains its maximum at a unique point.

Observe that

$$
T_{q}=\inf _{u \in E^{+} \backslash\{0\}} \max _{w \in E_{u}} \frac{a_{1}(w)}{|w|_{q}^{2}}=\inf _{u \in E^{+} \backslash\{0\}} \max _{w \in \hat{E}_{u}} \frac{a_{1}(w)}{|w|_{q}^{2}} .
$$

If the function

$$
m_{u}(w)=\frac{a_{1}(w)}{|w|_{q}^{2}}
$$

attains its maximum on $E_{u}$ at $w \in \hat{E}_{u}$, setting $\hat{w}=m_{u}(w)^{1 /(q-2)} w /|w|_{q}$, then $m_{u}(\hat{w})=m_{u}(w)$ and, for any $v \in E_{u}$,

$$
0=m_{u}^{\prime}(w) v=\frac{2 m_{u}(w)^{1 /(q-2)}}{|w|_{q}} \Re\left[a_{1}(\hat{w}, v)-\int_{\mathbb{R}^{3}}|\hat{w}|^{q-2} \hat{w} \bar{v}\right] .
$$

This implies that $\hat{w} \in \mathscr{M}_{q}$, consequently,

$$
\gamma_{q} \leq \Gamma_{q}(\hat{w})=\frac{q-2}{2 q}\left(m_{u}(\hat{w})\right)^{q /(q-2)}=\frac{q-2}{2 q}\left(m_{u}(w)\right)^{q /(q-2)},
$$

hence, $T_{q} \geq\left(\frac{2 q \gamma_{q}}{q-2}\right)^{(q-2) / q}$.

On the other hand, let $z$ be a least energy solution of (4.5), $z=z^{+}+z^{-}, z^{-}=$ $\mathscr{J}_{q}\left(z^{+}\right)$. Take $u=z^{+}$. One has $m_{u}(z)=|z|_{q}^{q-2}$. Plainly, one checks as above that, for $v \in E^{-}$,

$$
\psi_{u}^{\prime}\left(z^{-}\right) v=\frac{2}{|z|_{q}^{2}} \Re\left[a_{1}\left(z^{-}, v\right)-\int_{\mathbb{R}^{3}}|z|^{q-2} z \bar{v}\right]=0
$$


and

$$
\psi_{u}^{\prime \prime}\left(z^{-}\right)[v, v] \leq \frac{2}{|z|_{q}^{4}} \Re\left[a_{1}(v)|z|_{q}^{2}-a_{1}(z)|z|^{2-q} \int_{\mathbb{R}^{3}}|z|^{q-2}|v|^{2}\right] .
$$

Therefore,

$$
T_{q}\left(z^{+}\right)=\frac{a_{1}(z)}{|z|_{q}^{2}}=|z|_{q}^{q-2}=\left(\frac{2 q \gamma_{q}}{q-2}\right)^{(q-2) / q},
$$

completing the proof.

LEMMA 4.6. If $g(s)=c_{0} s^{q-2}$ and $\mu \leq 0$, then the corresponding least energy of (4.3) denoted by $\gamma_{\mu \nu}(q)$ satisfies

$$
\gamma_{\mu \nu}(q) \leq(a+\mu)^{\frac{2(3-q)}{q-2}}\left(c_{0} \nu\right)^{\frac{-2}{q-2}} \gamma_{q} .
$$

Proof. Observe that, setting $z(x)=u(x /(a+\mu))$, (4.3) is equivalent to

$$
-i \alpha \cdot \nabla z+\beta z+\frac{\mu}{a+\mu}(I-\beta) z=\frac{\nu}{a+\mu} g(|z|) z
$$

with energy functional defined by

$$
\Gamma_{\nu / \mu}(z):=\frac{1}{2} a_{1}(z)+\frac{\mu}{a+\mu}\left|z_{2}\right|_{2}^{2}-\frac{\nu}{a+\mu} \int_{\mathbb{R}^{3}} G(|z|)
$$

and the least energy denoted by $\gamma_{\nu / \mu}$, where $z=\left(z_{1}, z_{2}\right) \in \mathbb{C}^{2} \times \mathbb{C}^{2}$. One has

$$
\gamma_{\mu \nu}=(a+\mu)^{-2} \gamma_{\nu / \mu} .
$$

Now assume $g(s)=c_{0} s^{q-2}$ and $\mu \leq 0$. We denote by $\gamma_{\nu / \mu}(q)$ the least energy corresponding to (4.7). Let $z$ be a least energy solution of (4.5), $u=z^{+}$, and $e_{q} \in E_{u}$ with $\Gamma_{\nu / \mu}\left(e_{q}\right)=\max _{v \in E_{u}} \Gamma_{\nu / \mu}(v)$. Then by Lemma 4.5,

$$
\begin{aligned}
\gamma_{\nu / \mu}(q) & \leq \Gamma_{\nu / \mu}\left(e_{q}\right) \\
& =\frac{q-2}{2 q}\left(\frac{a+\mu}{c_{0} \nu}\right)^{\frac{2}{q-2}}\left(\frac{a_{1}\left(e_{q}\right)+\frac{2 \mu}{a+\mu}\left|e_{q 2}\right|_{2}^{2}}{\left|e_{q}\right|_{q}^{2}}\right)^{\frac{q}{q-2}} \\
& \leq \frac{q-2}{2 q}\left(\frac{a+\mu}{c_{0} \nu}\right)^{\frac{2}{q-2}} T_{q}^{\frac{q}{q-2}} \\
& =\left(\frac{a+\mu}{c_{0} \nu}\right)^{\frac{2}{q-2}} \gamma_{q} .
\end{aligned}
$$

This, jointly with (4.8), yields the desired conclusion (4.6).

4.2. The critical equation (4.1). We now turn to the critical problem. Define $\mathscr{J}_{\mu \nu}^{*}, J_{\mu \nu}^{*}$ and $\mathscr{M}_{\mu \nu}^{*}, \gamma_{\mu \nu}^{*}:=\inf _{\mathscr{M}_{q}} J_{\mu \nu}^{*}$ etc. as before.

Observe that, for $\mu \in \mathbb{R}$,

$$
\int_{\mathbb{R}^{3}}\left\langle\left(A_{a}+\mu\right) u, u^{+}-u^{-}\right\rangle \geq \begin{cases}\left.\left.\frac{a-\mu}{a}|| A_{a}\right|^{1 / 2} u\right|_{2} ^{2} & \text { if } \mu \geq 0 \\ \left.\left.\frac{a+\mu}{a}|| A_{a}\right|^{1 / 2} u\right|_{2} ^{2} & \text { if } \mu<0\end{cases}
$$


LEMMA 4.7. $\gamma_{\mu \nu}^{*}$ is attained if

$$
\gamma_{\mu \nu}^{*}<\ell^{*}:=\frac{S^{3 / 2}}{6 \nu^{2}}\left(\frac{a+\mu}{a}\right)^{3}
$$

Proof. Let $\left\{u_{n}\right\}$ be a $(P S)_{c}$ sequence with $c=\gamma_{\mu \nu}^{*}: \Gamma_{\mu \nu}^{*}\left(u_{n}\right) \rightarrow c$ and $\Gamma_{\mu \nu}^{*^{\prime}}\left(u_{n}\right) \rightarrow$ 0 as $n \rightarrow \infty$. It is not difficult to check that $\left\{u_{n}\right\}$ is bounded in $E$. By Lions' concentration principle [21], $\left\{u_{n}\right\}$ is either vanishing or non-vanishing.

Assume that $\left\{u_{n}\right\}$ is vanishing. Then $\left|u_{n}\right|_{s} \rightarrow 0$ for $s \in(2,3)$. By $\left(g_{1}\right),\left(g_{2}\right)$ one gets

$$
\Gamma_{\mu \nu}^{*}\left(u_{n}\right)-\frac{1}{3} \Gamma_{\mu \nu}^{*^{\prime}}\left(u_{n}\right) u_{n} \geq \frac{1}{6} \int_{\mathbb{R}^{3}}\left\langle\left(A_{a}+\mu\right) u_{n}, u_{n}\right\rangle+o(1)
$$

or

$$
\int_{\mathbb{R}^{3}}\left\langle\left(A_{a}+\mu\right) u_{n}, u_{n}\right\rangle \leq 6 c+o(1) .
$$

Similarly,

$$
\int_{\mathbb{R}^{3}}\left|u_{n}\right|^{3} \leq \frac{6 c}{\nu}+o(1)
$$

Moreover,

$$
\int_{\mathbb{R}^{3}}\left\langle\left(A_{a}+\mu\right) u_{n}, u_{n}^{+}-u_{n}^{-}\right\rangle-\nu \int_{\mathbb{R}^{3}}\left|u_{n}\right| u_{n}\left(u_{n}^{+}-u_{n}^{-}\right) \leq o(1) .
$$

Thus,

$$
|| A_{a}+\left.\left.\mu\right|^{1 / 2} u_{n}\right|_{2} ^{2} \leq \nu\left|u_{n}\right|_{3}^{2}\left|u_{n}^{+}-u_{n}^{-}\right|_{3}+o(1)
$$

which, together with (4.9) and Lemma 3.1, implies

$$
c \geq \ell^{*}=\frac{S^{3 / 2}}{6 \nu^{2}}\left(\frac{a+\mu}{a}\right)^{3}
$$

a contradiction.

Therefore, $\left\{u_{n}\right\}$ is non-vanishing, that is, there exist $r, \delta>0$ and $x_{n} \in \mathbb{R}^{3}$ such that, setting $v_{n}(x)=u_{n}\left(x+x_{n}\right)$, along a subsequence,

$$
\int_{B_{r}(0)}\left|v_{n}\right|^{2} \geq \delta
$$

Without loss of generality we assume $v_{n} \rightarrow v$. Then $v \neq 0$ and is a solution of (4.1). And so $\gamma_{\mu \nu}^{*}$ is attained.

Now the combination of Lemma 4.6 and Lemma 4.7 gives the proof of Proposition 4.1 .

Proof of Proposition 4.1. Observe that

$$
\gamma_{\mu \nu}^{*}<\gamma_{\mu \nu} \leq \gamma_{\mu \nu}(q)
$$


If

$$
(a+\mu)^{\frac{2(3-q)}{q-2}}\left(c_{0} \nu\right)^{\frac{-2}{q-2}} \gamma_{q}<\ell^{*},
$$

that is, (4.2) is satisfied, then $\gamma_{\mu \nu}^{*}<\ell^{*}$ so it is attained by Lemma 4.7.

As a consequence of Proposition 4.1 we see

LEMMA 4.8. If $\mu$ and $\nu$ satisfy (4.2) then

$$
\begin{aligned}
& \mathscr{L}_{\mu \nu}^{*}:=\left\{u \in E: \Gamma_{\mu \nu}^{*^{\prime}}(u)=0\right\} \neq \emptyset, \\
& \gamma_{\mu \nu}^{*}:=\inf \left\{\Gamma_{\mu \nu}^{*}(u): u \in \mathscr{L}_{\mu \nu}^{*} \backslash\{0\}\right\},
\end{aligned}
$$

and $\mathscr{R}_{\mu \nu}^{*}:=\left\{u \in \mathscr{L}_{a b}^{*}: \Gamma_{\mu \nu}^{*}(u)=\gamma_{\mu \nu}^{*},|u(0)|=|u|_{\infty}\right\}$ is compact in $E$.

Moreover, the following lemma is obvious.

Lemma 4.9. Let, for $j=1,2, \mu_{j} \in(-a, 0]$ and $\nu_{j}>0$.

i) If $\min \left\{\mu_{2}-\mu_{1}, \nu_{1}-\nu_{2}\right\} \geq 0$ then $\gamma_{\mu_{1} \nu_{1}}^{*} \leq \gamma_{\mu_{2} \nu_{2}}^{*}$.

2) Assume $\min \left\{\mu_{2}-\mu_{1}, \nu_{1}-\nu_{2}\right\}>0$, and either the pair $\left(\mu_{1}, \nu_{1}\right)$ or the pair $\left(\mu_{2}, \nu_{2}\right)$ satisfies $(4.2)$. Then $\gamma_{\mu_{1} \nu_{1}}^{*}<\gamma_{\mu_{2} \nu_{2}}^{*}$.

4.3. Auxiliary functionals. Assume that the sequence of functions $\hat{V}_{\varepsilon}$ and $\hat{W}_{\varepsilon} \in C \cap L^{\infty}\left(\mathbb{R}^{3}, \mathbb{R}\right), 0<\varepsilon \leq 1$, satisfy

$(\star) \varpi:=\sup _{\varepsilon, x}\left|\hat{V}_{\varepsilon}(x)\right|<a, \inf _{\varepsilon, x} \hat{W}_{\varepsilon}(x)>0 ; \hat{V}_{\varepsilon}(x) \rightarrow \mu$ and $\hat{W}_{\varepsilon}(x) \rightarrow \nu$ uniformly on bounded sets of $x$ as $\varepsilon \rightarrow 0$ with $\gamma_{\mu \nu}^{*}$ achieved (e.g. $\mu$ and $\nu$ satisfying (4.2)).

Consider the equation

$$
-i \alpha \cdot \nabla u+a \beta u+\hat{V}_{\varepsilon}(x) u=\hat{W}_{\varepsilon}(x)(g(|u|)+|u|) u
$$

for $u \in H^{1}\left(\mathbb{R}^{3}, \mathbb{C}^{4}\right)$. Denote

$$
\hat{\Phi}_{\varepsilon}(u)=\frac{1}{2}\left(\left\|u^{+}\right\|^{2}-\left\|u^{-}\right\|^{2}\right)+\frac{1}{2} \int_{\mathbb{R}^{3}} \hat{V}_{\varepsilon}(x)|u|^{2}-\int_{\mathbb{R}^{3}} \hat{W}_{\varepsilon}(x) F(|u|),
$$

As before, define the associated $\hat{c}_{\varepsilon}, \hat{\mathscr{N}}_{\varepsilon}$, etc.

Note that, setting $V_{\varepsilon}^{0}(x)=\hat{V}_{\varepsilon}(x)-\mu$ and $W_{\varepsilon}^{0}(x)=\nu-\hat{W}_{\varepsilon}(x)$, we have by definition

$$
\hat{\Phi}_{\varepsilon}(u)=\Gamma_{\mu \nu}^{*}(u)+\frac{1}{2} \int_{\mathbb{R}^{3}} V_{\varepsilon}^{0}(x)|u|^{2}+\int_{\mathbb{R}^{3}} W_{\varepsilon}^{0}(x) F(|u|) .
$$

LEMMA 4.10. $\limsup _{\varepsilon \rightarrow 0} \hat{c}_{\varepsilon} \leq \gamma_{\mu \nu}^{*}$.

Proof. In virtue of Lemma 4.8, let $u=u^{-}+u^{+} \in \mathscr{R}_{\mu \nu}^{*}$, a least energy solution of (4.1) and set $e=u^{+}$. It is clear that $e \in \mathscr{M}_{\mu \nu}^{*}, \mathscr{J}_{\mu \nu}^{*}(e)=u^{-}$and $J_{\mu \nu}^{*}(e)=\gamma_{\mu \nu}^{*}$. There is a unique $t_{\varepsilon}>0$ such that $t_{\varepsilon} e \in \hat{\mathscr{N}}_{\varepsilon}$. One has

$$
\hat{c}_{\varepsilon} \leq \hat{I}_{\varepsilon}\left(t_{\varepsilon} e\right) .
$$

It is clear that $\left\{t_{\varepsilon}\right\}$ is bounded, hence, without loss of generality we can assume $t_{\varepsilon} \rightarrow t_{0}$ as $\varepsilon \rightarrow 0$. 
Observe that (4.11) induces that

$$
\begin{aligned}
& \left(\hat{\Phi}_{\varepsilon}\left(t_{\varepsilon} e+\hat{h}_{\varepsilon}\left(t_{\varepsilon} e\right)\right)-\hat{\Phi}_{\varepsilon}\left(t_{\varepsilon} e+\mathscr{J}_{\mu \nu}^{*}\left(t_{\varepsilon} e\right)\right)\right) \\
& +\left(\Gamma_{\mu \nu}^{*}\left(t_{\varepsilon} e+\mathscr{J}_{\mu \nu}^{*}\left(t_{\varepsilon} e\right)\right)-\Gamma_{\mu \nu}^{*}\left(t_{\varepsilon} e+\hat{h}_{\varepsilon}\left(t_{\varepsilon} e\right)\right)\right) \\
= & \frac{1}{2} \int_{\mathbb{R}^{3}} V_{\varepsilon}^{0}(x)\left(\left|t_{\varepsilon} e+\hat{h}_{\varepsilon}\left(t_{\varepsilon} e\right)\right|^{2}-\left|t_{\varepsilon} e+\mathscr{J}_{\mu \nu}^{*}\left(t_{\varepsilon} e\right)\right|^{2}\right) \\
& +\int_{\mathbb{R}^{3}} W_{\varepsilon}^{0}(x)\left(F\left(\left|t_{\varepsilon} e+\hat{h}_{\varepsilon}\left(t_{\varepsilon} e\right)\right|\right)-F\left(\left|t_{\varepsilon} e+\mathscr{J}_{\mu \nu}^{*}\left(t_{\varepsilon} e\right)\right|\right)\right) .
\end{aligned}
$$

Since, denoting $z_{\varepsilon}=\mathscr{J}_{\mu \nu}^{*}\left(t_{\varepsilon} e\right)-\hat{h}_{\varepsilon}\left(t_{\varepsilon} e\right)$,

$$
\left|t_{\varepsilon} e+\hat{h}_{\varepsilon}\left(t_{\varepsilon} e\right)\right|^{2}-\left|t_{\varepsilon} e+\mathscr{J}_{\mu \nu}^{*}\left(t_{\varepsilon} e\right)\right|^{2}=\left|z_{\varepsilon}\right|^{2}-2 \Re\left\langle t_{\varepsilon} e+\mathscr{J}_{\mu \nu}^{*}\left(t_{\varepsilon} e\right), z_{\varepsilon}\right\rangle
$$

and

$$
\begin{aligned}
& F\left(\left|t_{\varepsilon} e+\hat{h}_{\varepsilon}\left(t_{\varepsilon} e\right)\right|\right)-F\left(\left|t_{\varepsilon} e+\mathscr{J}_{\mu \nu}^{*}\left(t_{\varepsilon} e\right)\right|\right) \\
= & F\left(\left|t_{\varepsilon} e+\mathscr{J}_{\mu \nu}^{*}\left(t_{\varepsilon} e\right)-z_{\varepsilon}\right|\right)-F\left(\left|t_{\varepsilon} e+\mathscr{J}_{\mu \nu}^{*}\left(t_{\varepsilon} e\right)\right|\right) \\
= & -f\left(\left|t_{\varepsilon} e+\mathscr{J}_{\mu \nu}^{*}\left(t_{\varepsilon} e\right)\right|\right) \Re\left\langle t_{\varepsilon} e+\mathscr{J}_{\mu \nu}^{*}\left(t_{\varepsilon} e\right), z_{\varepsilon}\right\rangle+K_{\varepsilon}(x)
\end{aligned}
$$

with

$$
\begin{aligned}
K_{\varepsilon}(x) & :=\int_{0}^{1}(1-s)\left(f\left(\left|t_{\varepsilon} e+\mathscr{J}_{\mu \nu}^{*}\left(t_{\varepsilon} e\right)-s z_{\varepsilon}\right|\right)\left|z_{\varepsilon}\right|^{2}\right. \\
& \left.+\frac{f^{\prime}\left(\left|t_{\varepsilon} e+\mathscr{J}_{\mu \nu}^{*}\left(t_{\varepsilon} e\right)-s z_{\varepsilon}\right|\right)}{\left|t_{\varepsilon} e+\mathscr{J}_{\mu \nu}^{*}\left(t_{\varepsilon} e\right)-s z_{\varepsilon}\right|}\left(\Re\left\langle t_{\varepsilon} e+\mathscr{J}_{\mu \nu}^{*}\left(t_{\varepsilon} e\right)-s z_{\varepsilon},-z_{\varepsilon}\right\rangle\right)^{2}\right) d s
\end{aligned}
$$

we get from (4.13) (remark that $W_{\varepsilon}^{0}(x) \leq \nu$ )

$$
\begin{aligned}
& \left(\hat{\Phi}_{\varepsilon}\left(t_{\varepsilon} e+\hat{h}_{\varepsilon}\left(t_{\varepsilon} e\right)\right)-\hat{\Phi}_{\varepsilon}\left(t_{\varepsilon} e+\mathscr{J}_{\mu \nu}^{*}\left(t_{\varepsilon} e\right)\right)\right) \\
& +\left(\Gamma_{\mu \nu}^{*}\left(t_{\varepsilon} e+\mathscr{J}_{\mu \nu}^{*}\left(t_{\varepsilon} e\right)\right)-\Gamma_{\mu \nu}^{*}\left(t_{\varepsilon} e+\hat{h}_{\varepsilon}\left(t_{\varepsilon} e\right)\right)\right) \\
\leq & \frac{1}{2} \int_{\mathbb{R}^{3}} V_{\varepsilon}^{0}(x)\left|z_{\varepsilon}\right|^{2}-\Re \int_{\mathbb{R}^{3}} V_{\varepsilon}^{0}(x)\left\langle t_{\varepsilon} e+\mathscr{J}_{\mu \nu}^{*}\left(t_{\varepsilon} e\right), z_{\varepsilon}\right\rangle \\
& -\Re \int_{\mathbb{R}^{3}} W_{\varepsilon}^{0}(x) f\left(\left|t_{\varepsilon} e+\mathscr{J}_{\mu \nu}^{*}\left(t_{\varepsilon} e\right)\right|\right)\left\langle t_{\varepsilon} e+\mathscr{J}_{\mu \nu}^{*}\left(t_{\varepsilon} e\right), z_{\varepsilon}\right\rangle \\
& +\nu \int_{\mathbb{R}^{3}} K_{\varepsilon}(x) .
\end{aligned}
$$

Remark that one has, similar to (4.4) (with $z$ replaced by $z_{\varepsilon}$ ),

$$
\begin{aligned}
& \Gamma_{\mu \nu}^{*}\left(t_{\varepsilon} e+\mathscr{J}_{\mu \nu}^{*}\left(t_{\varepsilon} e\right)\right)-\Gamma_{\mu \nu}^{*}\left(t_{\varepsilon} e+\hat{h}_{\varepsilon}\left(t_{\varepsilon} e\right)\right) \\
= & \frac{1}{2}\left(\left\|z_{\varepsilon}\right\|^{2}-\mu\left|z_{\varepsilon}\right|_{2}^{2}\right)+\nu \int_{\mathbb{R}^{3}} K_{\varepsilon}(x)
\end{aligned}
$$

and, by the representation (3.4) with $\Phi_{\varepsilon}$ replaced by $\hat{\Phi}_{\varepsilon}$,

$$
\hat{\Phi}_{\varepsilon}\left(t_{\varepsilon} e+\hat{h}_{\varepsilon}\left(t_{\varepsilon} e\right)\right)-\hat{\Phi}_{\varepsilon}\left(t_{\varepsilon} e+\mathscr{J}_{\mu \nu}^{*}\left(t_{\varepsilon} e\right)\right) \geq \frac{1}{2}\left(\left\|z_{\varepsilon}\right\|^{2}-\int_{\mathbb{R}^{3}} \hat{V}_{\varepsilon}(x)\left|z_{\varepsilon}\right|^{2}\right) .
$$


Thus (4.14) (jointly with $\left.\left(g_{1}\right)\right)$ implies

$$
\begin{aligned}
& \left\|z_{\varepsilon}\right\|^{2}-\varpi\left|z_{\varepsilon}\right|_{2}^{2} \\
\leq & -\Re \int_{\mathbb{R}^{3}} V_{\varepsilon}^{0}(x)\left\langle t_{\varepsilon} e+\mathscr{J}_{\mu \nu}^{*}\left(t_{\varepsilon} e\right), z_{\varepsilon}\right\rangle \\
& -\Re \int_{\mathbb{R}^{3}} W_{\varepsilon}^{0}(x) g\left(\left|t_{\varepsilon} e+\mathscr{J}_{\mu \nu}^{*}\left(t_{\varepsilon} e\right)\right|\right)\left\langle t_{\varepsilon} e+\mathscr{J}_{\mu \nu}^{*}\left(t_{\varepsilon} e\right), z_{\varepsilon}\right\rangle \\
\leq & \int_{\mathbb{R}^{3}}\left|V_{\varepsilon}^{0}(x)\right|\left|t_{\varepsilon} e+\mathscr{J}_{\mu \nu}^{*}\left(t_{\varepsilon} e\right)\right|\left|z_{\varepsilon}\right| \\
& +c_{1} \int_{\mathbb{R}^{3}}\left|W_{\varepsilon}^{0}(x)\right|\left|t_{\varepsilon} e+\mathscr{J}_{\mu \nu}^{*}\left(t_{\varepsilon} e\right)\right|\left|z_{\varepsilon}\right| \\
& +c_{1} \int_{\mathbb{R}^{3}}\left|W_{\varepsilon}^{0}(x)\right|\left|t_{\varepsilon} e+\mathscr{J}_{\mu \nu}^{*}\left(t_{\varepsilon} e\right)\right|^{p-1}\left|z_{\varepsilon}\right| \\
\leq & c_{2}\left(\int_{\mathbb{R}^{3}}\left(\left|V_{\varepsilon}^{0}(x)\right|+\left|W_{\varepsilon}^{0}(x)\right|\right)^{2}\left|t_{\varepsilon} e+\mathscr{J}_{\mu \nu}^{*}\left(t_{\varepsilon} e\right)\right|^{2}\right)^{1 / 2}\left|z_{\varepsilon}\right|_{2} \\
& +c_{1}\left(\int_{\mathbb{R}^{3}}\left|W_{\varepsilon}^{0}(x)\right|^{p /(p-1)}\left|t_{\varepsilon} e+\mathscr{J}_{\mu \nu}^{*}\left(t_{\varepsilon} e\right)\right|^{p}\right)^{(p-1) / p}\left|z_{\varepsilon}\right|_{p} .
\end{aligned}
$$

Since $t_{\varepsilon} \rightarrow t_{0}$ and $e$ is exponentially decaying, we have for $q=2, p$,

$$
\limsup _{R \rightarrow \infty} \int_{|x| \geq R}\left|t_{\varepsilon} e+\mathscr{J}_{\mu \nu}^{*}\left(t_{\varepsilon} e\right)\right|^{q}=0
$$

which implies that

$$
\begin{aligned}
& \int_{\mathbb{R}^{3}}\left(\left|V_{\varepsilon}^{0}(x)\right|+\left|W_{\varepsilon}^{0}(x)\right|\right)^{2}\left|t_{\varepsilon} e+\mathscr{J}_{\mu \nu}^{*}\left(t_{\varepsilon} e\right)\right|^{2} \\
= & \left(\int_{|x| \leq R}+\int_{|x|>R}\right)\left(\left|V_{\varepsilon}^{0}(x)\right|+\left|W_{\varepsilon}^{0}(x)\right|\right)^{2}\left|t_{\varepsilon} e+\mathscr{J}_{\mu \nu}^{*}\left(t_{\varepsilon} e\right)\right|^{2} \\
\leq & \int_{|x| \leq R}\left(\left|V_{\varepsilon}^{0}(x)\right|+\left|W_{\varepsilon}^{0}(x)\right|\right)^{2}\left|t_{\varepsilon} e+\mathscr{J}_{\mu \nu}^{*}\left(t_{\varepsilon} e\right)\right|^{2} \\
& +c_{3} \int_{|x|>R}\left|t_{\varepsilon} e+\mathscr{J}_{\mu \nu}^{*}\left(t_{\varepsilon} e\right)\right|^{2} \\
= & o(1)
\end{aligned}
$$

as $\varepsilon \rightarrow 0$, and similarly

$$
\int_{\mathbb{R}^{3}}\left|W_{\varepsilon}^{0}(x)\right|^{p /(p-1)}\left|t_{\varepsilon} e+\mathscr{J}_{\mu \nu}^{*}\left(t_{\varepsilon} e\right)\right|^{p}=o(1)
$$

as $\varepsilon \rightarrow 0$. Thus, since $\varpi<a$ by the assumption $(*)$, it follows from (4.16) that $\left\|z_{\varepsilon}\right\|=\left\|\hat{h}_{\varepsilon}\left(t_{\varepsilon} e\right)-\mathscr{J}_{\mu \nu}^{*}\left(t_{\varepsilon} e\right)\right\| \rightarrow 0$, that is, $\hat{h}_{\varepsilon}\left(t_{\varepsilon} e\right) \rightarrow \mathscr{J}_{\mu \nu}^{*}\left(t_{0} e\right)$. Consequently,

$$
\int_{\mathbb{R}^{3}} V_{\varepsilon}^{0}(x)\left|t_{\varepsilon} e+\hat{h}_{\varepsilon}\left(t_{\varepsilon} e\right)\right|^{2} \rightarrow 0 \quad \text { and } \quad \int_{\mathbb{R}^{3}} W_{\varepsilon}^{0}(x) F\left(\left|t_{\varepsilon} e+\hat{h}_{\varepsilon}\left(t_{\varepsilon} e\right)\right|\right) \rightarrow 0
$$

as $\varepsilon \rightarrow 0$. This, jointly with (4.11), implies

$$
\begin{aligned}
\hat{\Phi}_{\varepsilon}\left(t_{\varepsilon} e+\hat{h}_{\varepsilon}\left(t_{\varepsilon} e\right)\right) & =\Gamma_{\mu \nu}^{*}\left(t_{\varepsilon} e+\hat{h}_{\varepsilon}\left(t_{\varepsilon} e\right)\right)+o(1) \\
& =\Gamma_{\mu \nu}^{*}\left(t_{0} e+\mathscr{J}_{\mu \nu}^{*}\left(t_{0} e\right)\right)+o(1),
\end{aligned}
$$


that is,

$$
\hat{I}_{\varepsilon}\left(t_{\varepsilon} e\right)=J_{\mu \nu}^{*}\left(t_{0} e\right)+o(1)
$$

as $\varepsilon \rightarrow 0$. Recalling that by Lemma 4.3

$$
J_{\mu \nu}^{*}\left(t_{0} e\right) \leq \max _{v \in E_{e}} \Gamma_{\mu \nu}^{*}(v)=J_{\mu \nu}^{*}(e)=\gamma_{\mu \nu}^{*},
$$

we obtain, jointly with (4.12),

$$
\lim _{\varepsilon \rightarrow 0} \hat{c}_{\varepsilon} \leq \lim _{\varepsilon \rightarrow 0} \hat{I}_{\varepsilon}\left(t_{\varepsilon} e\right)=J_{\mu \nu}^{*}\left(t_{0} e\right) \leq \gamma_{\mu \nu}^{*}
$$

as claimed.

Below, for $\mu \in\left[\tau, \tau_{\infty}\right]$ and $\nu \in\left[\pi_{\infty}, \pi\right]$, we set

$$
\begin{aligned}
& V^{\mu}(x):=\max \{\mu, V(x)\}, \\
& W^{\nu}(x):=\min \{\nu, W(x)\},
\end{aligned}
$$

and let $V_{\varepsilon}^{\mu}(x)=V^{\mu}(\varepsilon x), W_{\varepsilon}^{\nu}(x)=W^{\nu}(\varepsilon x)$. Consider the functional

$$
\Phi_{\varepsilon}^{\mu \nu}(u)=\frac{1}{2}\left(\left\|u^{+}\right\|^{2}-\left\|u^{-}\right\|^{2}\right)+\frac{1}{2} \int_{\mathbb{R}^{3}} V_{\varepsilon}^{\mu}(x)|u|^{2}-\int_{\mathbb{R}^{3}} W_{\varepsilon}^{\nu}(x) F(|u|)
$$

with $\mathscr{N}_{\varepsilon}^{\mu \nu}, c_{\varepsilon}^{\mu \nu}$ and so on as before. By definition and Lemma 4.9,

$$
\gamma_{\tau \pi}^{*} \leq \gamma_{V(0) W(0)}^{*} \leq \gamma_{V^{\mu}(0) W^{\nu}(0)}^{*} .
$$

Moreover, observe that

$$
\Phi_{\varepsilon}^{\mu \nu}(u)=\Gamma_{\mu \nu}^{*}(u)+\frac{1}{2} \int_{\mathbb{R}^{3}}\left(V_{\varepsilon}^{\mu}(x)-\mu\right)|u|^{2}+\int_{\mathbb{R}^{3}}\left(\nu-W_{\varepsilon}^{\nu}(x)\right) F(|u|) .
$$

This, together with Lemma 4.10, shows that if $\mu$ and $\nu$ satisfy (4.2) then

$$
\gamma_{\mu \nu}^{*} \leq c_{\varepsilon}^{\mu \nu} \quad \text { and } \quad \limsup _{\varepsilon \rightarrow 0} c_{\varepsilon}^{\mu \nu} \leq \gamma_{V^{\mu}(0) W^{\nu}(0)}^{*}
$$

and particularly

$$
\lim _{\varepsilon \rightarrow 0} c_{\varepsilon}^{\mu \nu}=\gamma_{\mu \nu}^{*} \quad \text { if } V(0) \leq \mu \text { and } W(0) \geq \nu .
$$

5. Proofs of the main results. We are now giving the proofs of the main results on the critical equation:

$$
-i \alpha \cdot \nabla u+a \beta u+V_{\varepsilon}(x) u=W_{\varepsilon}(x) f(|u|) u, \quad u \in H^{1}\left(\mathbb{R}^{3}, \mathbb{C}^{4}\right)
$$

where $f(|u|)=g(|u|)+|u|$ with $g$ satisfying $\left(g_{1}\right)$ and $\left(g_{2}\right)$. Recall that, by assumption, $V(x) \leq 0$ hence $|V|_{\infty}=-\tau$. Observe that, by $\left(\hat{P}_{0}\right)$ and $(4.2)$,

$$
\gamma_{\mu \nu}^{*}<\ell^{*}, \text { for } \nu \in\left[\pi_{\infty}, \pi\right] \text { and } \mu \leq \tau_{\infty} \text { sufficiently close to } \tau_{\infty} .
$$

Note also that, for any $x_{0} \in \mathbb{R}^{3}$, setting $\tilde{V}(x)=V\left(x+x_{0}\right)$ and $\tilde{W}(x)=W\left(x+x_{0}\right)$, if $\tilde{w}(x)$ is a solution of

$$
-i \varepsilon \alpha \cdot \nabla \tilde{w}+a \beta \tilde{w}+\tilde{V}(x) \tilde{w}=\tilde{W}(x) f(|\tilde{w}|) \tilde{w},
$$

then $w(x):=\tilde{w}\left(x-x_{0}\right)$ solves $(5.1)$. 
5.1. Proof of Theorem 2.2. Assume $\left(P_{0}\right)-\left(\hat{P}_{0}\right)$ and $\left(P_{1}\right)$ are satisfied. By virtue of the above observation, without loss of generality, we can assume that $0 \in \mathscr{W}$ such that $V(0)=\min _{x \in \mathscr{W}} V(x)$. Then $\pi=W(0)$ and $\kappa:=V(0) \leq \tau_{\infty}$. Consider the functional $\Phi_{\varepsilon}$.

LEMma 5.1. Assume $\left(P_{0}\right)-\left(\hat{P}_{0}\right)$ and $\left(P_{1}\right)$ are satisfied. Then $c_{\varepsilon}$ is attained for small $\varepsilon$.

Proof. Given $\varepsilon>0$, let $u_{k} \in \mathscr{N}_{\varepsilon}$ be a minimizing sequence: $I_{\varepsilon}\left(u_{k}\right) \rightarrow c_{\varepsilon}$. By the Ekeland variational principle we can assume that $u_{k}$ is, in addition, a $(P S)_{c_{\varepsilon}}$ sequence for $I_{\varepsilon}$ on $\mathscr{N}_{\varepsilon}$. A standard argument shows that $u_{k}$ is in fact a $(P S)_{c_{\varepsilon}}$ sequence for $I_{\varepsilon}$ on $E^{+}$(see, e.g., $[23,30]$ ). Then $w_{k}=u_{k}+h_{\varepsilon}\left(u_{k}\right)$ is a $(P S)_{c_{\varepsilon}}$ sequence for $\Phi_{\varepsilon}$ on $E: \Phi_{\varepsilon}\left(w_{k}\right) \rightarrow c_{\varepsilon}$ and $\Phi_{\varepsilon}^{\prime}\left(w_{k}\right) \rightarrow 0$ as $k \rightarrow \infty$. It is easy to see that $\left\{w_{k}\right\}$ is bounded in $E$. We can assume without loss of generality that $w_{k} \rightarrow w_{\varepsilon} \in \mathscr{K}_{\varepsilon}$ in $E$. If $w_{\varepsilon} \neq 0$ then clearly $\Phi_{\varepsilon}\left(w_{\varepsilon}\right)=c_{\varepsilon}$. So we are going to check that $w_{\varepsilon} \neq 0$ for all $\varepsilon>0$ small.

Assume by contradiction that there is a sequence $\varepsilon_{j} \rightarrow 0$ with $w_{\varepsilon_{j}}=0$. Then $w_{k} \rightarrow 0$ in $E$ and $L^{s}$ for $s \in[2,3], w_{k} \rightarrow 0$ in $L_{l o c}^{s}$ for $s \in(1,3)$, and $w_{k}(x) \rightarrow 0$ a.e. in $x \in \mathbb{R}^{3}$. Observe that $\tau_{\infty} \geq \kappa$ by $\left(P_{1}\right)$. By $\left(\hat{P}_{0}\right)$, one can choose $\kappa \leq \mu \leq \tau_{\infty}$ satisfying

$$
\left(\frac{a+\mu}{a}\right)^{12-5 q}\left(\frac{a}{\nu}\right)^{2(3-q)}<\mathcal{R}_{q}
$$

for any $\nu \in\left(\pi_{\infty}, \pi\right)$. Consider the functional $\Phi_{\varepsilon_{j}}^{\mu \nu}$. Remark that $V_{\varepsilon}^{\mu}(0)=\mu$ and $W_{\varepsilon}^{\nu}(0)=\nu$, thus

$$
\gamma_{\mu \nu}^{*}=\lim _{\varepsilon_{j} \rightarrow 0} c_{\varepsilon_{j}}^{\mu \nu}
$$

by (4.19). Let $t_{k}>0$ be such that $t_{k} u_{k} \in \mathscr{N}_{\varepsilon_{j}}^{\mu \nu}$. Then $\left\{t_{k}\right\}$ is bounded and we may assume $t_{k} \rightarrow t_{0}$ as $k \rightarrow \infty$. By $\left(P_{1}\right)$, the set $O_{\varepsilon}:=\left\{x \in \mathbb{R}^{3}: V_{\varepsilon}(x)<\mu\right.$ or $\left.W_{\varepsilon}(x) \geq \nu\right\}$ is bounded. Remark that $\Phi_{\varepsilon_{j}}\left(t_{k} u_{k}+h_{\varepsilon_{j}}^{\mu \nu}\left(t_{k} u_{k}\right)\right) \leq I_{\varepsilon_{j}}\left(u_{k}\right)$. We obtain

$$
\begin{aligned}
c_{\varepsilon_{j}}^{\mu \nu} \leq & I_{\varepsilon_{j}}^{\mu \nu}\left(t_{k} u_{k}\right)=\Phi_{\varepsilon_{j}}^{\mu \nu}\left(t_{k} u_{k}+h_{\varepsilon_{j}}^{\mu \nu}\left(t_{k} u_{k}\right)\right) \\
= & \Phi_{\varepsilon_{j}}\left(t_{k} u_{k}+h_{\varepsilon_{j}}^{\mu \nu}\left(t_{k} u_{k}\right)\right)+\frac{1}{2} \int_{\mathbb{R}^{3}}\left(V_{\varepsilon_{j}}^{\mu}(x)-V_{\varepsilon_{j}}(x)\right)\left|t_{k} u_{k}+h_{\varepsilon_{j}}^{\mu \nu}\left(t_{k} u_{k}\right)\right|^{2} \\
& \quad+\int_{\mathbb{R}^{3}}\left(W_{\varepsilon_{j}}(x)-W_{\varepsilon_{j}}^{\nu}(x)\right) F\left(\left|t_{k} u_{k}+h_{\varepsilon_{j}}^{\mu \nu}\left(t_{k} u_{k}\right)\right|\right) \\
\leq & I_{\varepsilon_{j}}\left(u_{k}\right)+\frac{1}{2} \int_{O_{\varepsilon_{j}}}\left(\mu-V_{\varepsilon_{j}}(x)\right)\left|t_{k} u_{k}+h_{\varepsilon_{j}}^{\mu \nu}\left(t_{k} u_{k}\right)\right|^{2} \\
& +\int_{O_{\varepsilon_{j}}}\left(W_{\varepsilon_{j}}(x)-\nu\right) F\left(\left|t_{k} u_{k}+h_{\varepsilon_{j}}^{\mu \nu}\left(t_{k} u_{k}\right)\right|\right) \\
= & c_{\varepsilon_{j}}+o(1)
\end{aligned}
$$

as $k \rightarrow \infty$, hence,

$$
c_{\varepsilon_{j}}^{\mu \nu} \leq c_{\varepsilon_{j}}
$$

On the other hand, let $v_{k} \in \mathscr{N}_{\varepsilon_{j}}^{\mu \nu}$ be a minimizing sequence for $I_{\varepsilon_{j}}^{\mu \nu}$ and set $z_{k}=$ $v_{k}+h_{\varepsilon_{j}}^{\mu \nu}\left(v_{k}\right)$. Denoting $E_{k}=E^{-} \oplus \mathbb{R}^{+} v_{k}$, since $V^{\mu} \geq V$ and $W^{\nu} \leq W$, one has

$$
I_{\varepsilon_{j}}^{\mu \nu}\left(v_{k}\right)=\max _{z \in E_{k}} \Phi_{\varepsilon_{j}}^{\mu \nu}(z) \geq \max _{z \in E_{k}} \Phi_{\varepsilon_{j}}(z) .
$$


Consequently, $c_{\varepsilon_{j}} \leq c_{\varepsilon_{j}}^{\mu \nu}$. This, together with (5.4) and (5.5), yields

$$
\gamma_{\mu \nu}^{*}=\lim _{\varepsilon_{j} \rightarrow 0} c_{\varepsilon_{j}}^{\mu \nu}=\lim _{\varepsilon_{j} \rightarrow 0} c_{\varepsilon_{j}}
$$

Now, since $\pi_{\infty}<\pi$, we can take $\pi_{\infty}<\nu_{1}<\nu_{2}<\pi$. We see by Lemma 4.9 and (5.6) the following

$$
\gamma_{\mu \nu_{1}}^{*}<\gamma_{\mu \nu_{2}}^{*}=\lim _{\varepsilon_{j} \rightarrow 0} c_{\varepsilon_{j}}=\gamma_{\mu \nu_{1}}^{*}
$$

a contradiction.

Remark that, by Lemma 3.9, the critical point $u_{\varepsilon}$ corresponding to $c_{\varepsilon}$ satisfies $u_{\varepsilon} \in \cap_{s \geq 2} W^{1, s}$.

From the last argument of the proof of Lemma 5.1 (just below (5.5)), we see also the following

LEMMA 5.2. $\lim \sup _{\varepsilon \rightarrow 0} c_{\varepsilon} \leq \limsup _{\varepsilon \rightarrow 0} c_{\varepsilon}^{\mu \pi}=\gamma_{\mu \pi}^{*}$ for any $\mu \geq \kappa$ satisfying (5.3) with $\nu=\pi$.

REMARK 5.3. It is not difficult to check that $\mathscr{S}_{\varepsilon}$ is compact for all small $\varepsilon>0$. Indeed, assume by contraction that, for some $\varepsilon_{j} \rightarrow 0, \mathscr{S}_{\varepsilon_{j}}$ is not compact in E. Let $u_{n}^{j} \in \mathscr{S}_{\varepsilon_{j}}$ with $u_{n}^{j} \rightarrow 0$ as $n \rightarrow \infty$. As done in proving the above Lemma 5.1 , one gets a contradiction.

For the later use, letting $D=-i \alpha \cdot \nabla$, we write (5.1) as

$$
D u=-a \beta u-V_{\varepsilon}(x) u+W_{\varepsilon}(x) f(|u|) u .
$$

By Lemma 3.9, $u \in \cap_{s \geq 2} W^{1, s}$ for any $u \in \mathscr{K}_{\varepsilon}$. Acting the operator $D$ on the two sides of the above representation and noting that $D^{2}=-\Delta$ we get

$$
\Delta u=\left(a^{2}-V_{\varepsilon}^{2}(x)\right) u+r_{\varepsilon}(x,|u|) u
$$

where

$$
\begin{aligned}
r_{\varepsilon}(x,|u|):= & D V_{\varepsilon}(x)+2 V_{\varepsilon}(x) W_{\varepsilon}(x) f(|u|)-W_{\varepsilon}(x)^{2} f(|u|)^{2} \\
& -\left(D W_{\varepsilon}(x) f(|u|)+\frac{f^{\prime}(|u|)}{|u|} W_{\varepsilon}(x) \Re\langle u, D u\rangle\right)
\end{aligned}
$$

Letting

$$
\operatorname{sgn} u=\left\{\begin{aligned}
\frac{\bar{u}}{|u|} & \text { if } u \neq 0 \\
0 & \text { if } u=0
\end{aligned}\right.
$$

by Kato's inequality ([11]), there holds

$$
\Delta|u| \geq \Re[\Delta u(\operatorname{sgn} u)]
$$

Observe that

$$
\Re\left[D V_{\varepsilon}(x) u \frac{\bar{u}}{|u|}\right]=0
$$

and

$$
\Re\left[\left(D W_{\varepsilon}(x) f(|u|)+\frac{f^{\prime}(|u|)}{|u|} W_{\varepsilon}(x) \Re\langle u, D u\rangle\right) u \frac{\bar{u}}{|u|}\right]=0 .
$$


Hence

$$
\Re\left[r_{\varepsilon}(x,|u|) u \frac{\bar{u}}{|u|}\right]=\left(2 V_{\varepsilon}(x)-W_{\varepsilon}(x) f(|u|)\right) W_{\varepsilon}(x) f(|u|)|u| .
$$

We obtain

$$
\Delta|u| \geq\left(a^{2}-V_{\varepsilon}^{2}(x)\right)|u|+\left(2 V_{\varepsilon}(x)-W_{\varepsilon}(x) f(|u|)\right) W_{\varepsilon}(x) f(|u|)|u| .
$$

This, together with (3.6), implies in particular that there is $\Lambda>0$ satisfying

$$
\Delta|u| \geq-\Lambda|u|
$$

It then follows from the sub-solution estimate $[20,27]$ that

$$
|u(x)| \leq C_{0} \int_{B_{1}(x)}|u(y)| d y
$$

with $C_{0}$ independent of $x$ and $u \in \mathscr{K}_{\varepsilon}, \varepsilon>0$, where $B_{1}(x)=\left\{y \in \mathbb{R}^{3}:|y| \leq 1\right\}$.

Now we turn to the concentration. Thus assume that $\left(P_{3}\right)$ also holds. Thus, by Proposition $4.1, \gamma_{\tau \pi}^{*}$ is attained.

LEMmA 5.4. Assume additionally that $\left(P_{3}\right)$ also holds. Let $u_{\varepsilon} \in S_{\varepsilon}$. There is a maximum point $y_{\varepsilon}$ of $\left|u_{\varepsilon}\right|$ such that $\lim _{\varepsilon \rightarrow 0} \operatorname{dist}\left(\varepsilon y_{\varepsilon}, \mathscr{A}_{w}\right)=0$, and for any sequence $\varepsilon y_{\varepsilon} \rightarrow y_{0}, v_{\varepsilon}(x):=u_{\varepsilon}\left(x+y_{\varepsilon}\right)$ converges in $H^{1}$ to a least energy solution of

$$
-i \alpha \cdot \nabla v+a \beta v+V\left(y_{0}\right) v=W\left(y_{0}\right) f(|v|) v .
$$

Proof. The proof will be carried out in several steps.

Step 1) Given arbitrarily a sequence $\varepsilon_{j} \rightarrow 0$ as $j \rightarrow \infty$, let $u_{j} \in \mathscr{S}_{j} \equiv \mathscr{S}_{\varepsilon_{j}}$. Then $\left\{u_{j}\right\}$ is bounded. A concentration argument shows that $\left\{u_{j}\right\}$ is either varnishing or non-varnishing.

If $\left\{u_{j}\right\}$ is varnishing then $\left|u_{j}\right|_{s} \rightarrow 0$ for $s \in(2,3)$. Note that, by Lemma 5.2, $\lim \sup _{j \rightarrow \infty} c_{\varepsilon_{j}} \leq \gamma_{\mu \pi}^{*}$ for $\mu$ close sufficiently to $\tau_{\infty}$. Recall that (see Proposition 4.1 and Lemma 4.7)

$$
\gamma_{\mu \pi}^{*}<\frac{S^{3 / 2}}{6 \pi^{2}}\left(\frac{a+\mu}{a}\right)^{3}<\frac{S^{3 / 2}}{6 \pi_{\infty}^{2}}\left(\frac{a+\tau_{\infty}}{a}\right)^{3} .
$$

It is not difficult to check that As before (see Lemma 4.7) that $\Phi_{\varepsilon_{j}}^{\infty}\left(u_{j}\right):=\Phi_{\varepsilon_{j}}^{\tau_{\infty} \pi_{\infty}}\left(u_{j}\right) \rightarrow$ $c$ (some $\left.c \leq \gamma_{\mu \pi}^{*}\right)$, and $\Phi_{\varepsilon_{j}}^{\infty^{\prime}}\left(u_{j}\right) \rightarrow 0$. By $\left(g_{1}\right)$ and $\left(g_{2}\right)$ one gets

$$
\int_{\mathbb{R}^{3}}\left\langle\left(A_{a}+V_{\varepsilon_{j}}^{\tau_{\infty}}\right) u_{j}, u_{j}\right\rangle \leq 6 c_{\varepsilon_{j}}+o(1)
$$

and

$$
\int_{\mathbb{R}^{3}} W_{\varepsilon_{j}}^{\pi_{\infty}}(x)\left|u_{j}\right|^{3} \leq 6 c_{\varepsilon_{j}}+o(1)
$$

Moreover,

$$
\int_{\mathbb{R}^{3}}\left\langle\left(A_{a}+V_{\varepsilon_{j}}^{\tau_{\infty}}\right) u_{j}, u_{j}\right\rangle-\int_{\mathbb{R}^{3}} W_{\varepsilon_{j}}^{\pi_{\infty}}(x)\left|u_{j}\right|^{3} \leq o(1) .
$$


Then (recalling that $V(x) \leq 0$ hence $\left|V_{\varepsilon_{j}}^{\tau_{\infty}}\right|_{\infty}=-\tau_{\infty}$ )

$$
\begin{aligned}
\frac{a+\tau_{\infty}}{a}\left\|u_{j}\right\|^{2} & \leq \int_{\mathbb{R}^{3}}\left\langle\left(A_{a}+V_{\varepsilon_{j}}^{\tau_{\infty}}\right) u_{j}, u_{j}^{+}-u_{j}^{-}\right\rangle \\
& \leq\left(6 c_{\varepsilon_{j}}\right)^{1 / 3} \pi_{\infty}^{2 / 3} S^{-1 / 2}\left\|u_{j}\right\|^{2}+o(1),
\end{aligned}
$$

that is,

$$
c \geq \frac{S^{3 / 2}}{6 \pi_{\infty}^{2}}\left(\frac{a+\tau_{\infty}}{a}\right)^{3}
$$

contradicting to $(5.10)$.

Therefore $\left\{u_{j}\right\}$ is non-varnishing, that is, there exist a sequence $\left\{y_{j}^{\prime}\right\} \subset \mathbb{R}^{3}$ and constants $r>0, \delta>0$ such that

$$
\liminf _{j \rightarrow \infty} \int_{B_{r}\left(y_{j}^{\prime}\right)}\left|u_{j}\right|^{2} \geq \delta
$$

Set

$$
v_{j}(x)=u_{j}\left(x+y_{j}^{\prime}\right)
$$

Then $v_{j}$ solves, denoting $\hat{V}_{\varepsilon_{j}}(x)=V\left(\varepsilon_{j}\left(x+y_{j}^{\prime}\right)\right)$ and $\hat{W}_{\varepsilon_{j}}(x)=W\left(\varepsilon_{j}\left(x+y_{j}^{\prime}\right)\right)$,

$$
-i \alpha \cdot \nabla v_{j}+a \beta v_{j}+\hat{V}_{\varepsilon_{j}}(x) v_{j}=\hat{W}_{\varepsilon_{j}}(x) f\left(\left|v_{j}\right|\right) v_{j}
$$

with least energy (using the notations of the previous section)

$$
\begin{aligned}
\hat{c}_{\varepsilon_{j}} & =\hat{\Phi}_{\varepsilon_{j}}\left(v_{j}\right) \\
& :=\frac{1}{2} \int_{\mathbb{R}^{3}}\left\langle A_{a} v_{j}, v_{j}\right\rangle+\hat{V}_{\varepsilon_{j}}(x)\left|v_{j}\right|^{2}-\int_{\mathbb{R}^{3}} \hat{W}_{\varepsilon_{j}}(x) F\left(\left|v_{j}\right|\right) \\
& =\int_{\mathbb{R}^{3}} \hat{W}_{\varepsilon_{j}}(x) \bar{F}\left(\left|v_{j}\right|\right)
\end{aligned}
$$

where $\bar{F}(|u|)=\frac{1}{2} f(|u|)|u|^{2}-F(|u|)$. Plainly,

$$
\hat{c}_{\varepsilon_{j}}=\hat{\Phi}_{\varepsilon_{j}}\left(v_{j}\right)=\Phi_{\varepsilon_{j}}\left(u_{j}\right)=c_{\varepsilon_{j}} .
$$

Additionally, $v_{j} \rightarrow u \neq 0$ in $E$ and $v_{j} \rightarrow u$ in $L_{l o c}^{s}$ for $s \in[1,3)$.

Since $V$ and $W$ are bounded, we can assume without loss of generality that $V\left(\varepsilon_{j} y_{j}^{\prime}\right) \rightarrow V_{0}$ and $W\left(\varepsilon_{j} y_{j}^{\prime}\right) \rightarrow W_{0}$ as $j \rightarrow \infty$. Since $\nabla V$ is bounded, one sees that, given arbitrarily $r>0$, for any $x \in B_{r}(0)$,

$$
\left|V\left(\varepsilon_{j} x+\varepsilon_{j} y_{j}^{\prime}\right)-V\left(\varepsilon_{j} y_{j}^{\prime}\right)\right|=\left|\int_{0}^{1} \nabla V\left(\varepsilon_{j} y_{j}^{\prime}+s \varepsilon_{j} x\right) \varepsilon_{j} x d s\right| \leq|\nabla V|_{\infty} r \varepsilon_{j} .
$$

This implies that $\hat{V}_{\varepsilon_{j}}(x) \rightarrow V_{0}$ as $j \rightarrow \infty$ uniformly on bounded sets of $x$. Similarly, $\hat{W}_{\varepsilon_{j}}(x) \rightarrow W_{0}$ as $j \rightarrow \infty$ uniformly on bounded sets of $x$. It then follows from (5.11) that, for any $\varphi \in C_{0}^{\infty}$,

$$
\begin{aligned}
0 & =\lim _{j \rightarrow \infty} \int_{\mathbb{R}^{3}}\left\langle\left(A_{a}+\hat{V}_{\varepsilon_{j}}(x)\right) v_{j}-\hat{W}_{\varepsilon_{j}}(x) f\left(\left|v_{j}\right|\right) v_{j}, \varphi\right\rangle \\
& =\int_{\mathbb{R}^{3}}\left\langle\left(A_{a}+V_{0}\right) u-W_{0} f(|u|) u, \varphi\right\rangle,
\end{aligned}
$$


Thus $u$ solves

$$
-i \alpha \cdot \nabla u+a \beta u+V_{0} u=W_{0} f(|u|) u
$$

with the energy

$$
\begin{aligned}
\Gamma_{V_{0} W_{0}}^{*}(u) & :=\frac{1}{2} \int_{\mathbb{R}^{3}}\left\langle A_{a} u, u\right\rangle+V_{0}|u|^{2}-\int_{\mathbb{R}^{3}} W_{0} F(|u|) \\
& =\int_{\mathbb{R}^{3}} W_{0} \bar{F}(|u|) \geq \gamma_{V_{0} W_{0}}^{*} .
\end{aligned}
$$

By a Fatou's lemma,

$$
\int_{\mathbb{R}^{3}} W_{0} \bar{F}(|u|) \leq \lim _{j \rightarrow \infty} \int_{\mathbb{R}^{3}} \hat{W}_{\varepsilon_{j}}(x) \bar{F}\left(\left|v_{j}\right|\right)
$$

which implies

$$
\Gamma_{V_{0} W_{0}}^{*}(u) \leq \lim _{j \rightarrow \infty} c_{\varepsilon_{j}} \leq \gamma_{V_{0} W_{0}}^{*}
$$

Therefore,

$$
\lim _{j \rightarrow \infty} c_{\varepsilon_{j}}=\Gamma_{V_{0} W_{0}}^{*}(u)=\gamma_{V_{0} W_{0}}^{*}
$$

and

$$
\lim _{j \rightarrow \infty} \int_{\mathbb{R}^{3}} \hat{W}_{\varepsilon_{j}}(x) \bar{F}\left(\left|v_{j}\right|\right)=\int_{\mathbb{R}^{3}} W_{0} \bar{F}(|u|)=\gamma_{V_{0} W_{0}}^{*}
$$

Let $\eta:[0, \infty) \rightarrow[0,1]$ be a smooth function satisfying $\eta(s)=1$ if $s \leq 1, \eta(s)=0$ if $s \geq 2$. Define $\tilde{v}_{j}(x)=\eta(2|x| / j) u(x)$. One has

$$
\left\|u-\tilde{v}_{j}\right\| \rightarrow 0 \text { and }\left|u-\tilde{v}_{j}\right|_{s} \rightarrow 0 \text { as } j \rightarrow \infty
$$

for $s \in[2,3]$. Setting $z_{j}=v_{j}-\tilde{v}_{j}$, one checks easily that, along a subsequence,

$$
\lim _{j \rightarrow \infty}\left|\int_{\mathbb{R}^{3}} \hat{W}_{\varepsilon_{j}}(x)\left(F\left(\left|v_{j}\right|\right)-F\left(\left|z_{j}\right|\right)-F\left(\left|\tilde{v}_{j}\right|\right)\right)\right|=0
$$

and

$$
\lim _{j \rightarrow \infty}\left|\int_{\mathbb{R}^{3}} \hat{W}_{\varepsilon_{j}}(x)\left(f\left(\left|v_{j}\right|\right) v_{j}-f\left(\left|z_{j}\right|\right) z_{j}-f\left(\left|\tilde{v}_{j}\right|\right) \tilde{v}_{j}\right) \bar{\varphi}\right|=0
$$

uniformly in $\varphi \in E$ with $\|\varphi\| \leq 1$ (see $[2,13,30]$ ). Using the exponentially decay of $u$, (5.14), and the facts that $\hat{V}_{\varepsilon_{j}}(x) \rightarrow V_{0}, \hat{W}_{\varepsilon_{j}}(x) \rightarrow W_{0}$ as $j \rightarrow \infty$ uniformly on any bounded set of $x$, one checks easily the following

$$
\begin{aligned}
\int_{\mathbb{R}^{3}} \hat{V}_{\varepsilon_{j}}(x)\left\langle v_{j}, \tilde{v}_{j}\right\rangle & \rightarrow \int_{\mathbb{R}^{3}} V_{0}|u|^{2} ; \\
\int_{\mathbb{R}^{3}} \hat{W}_{\varepsilon_{j}}(x) F\left(\left|\tilde{v}_{j}\right|\right) & \rightarrow \int_{\mathbb{R}^{3}} W_{0} F(|u|),
\end{aligned}
$$


Consequently, by (5.15),

$$
\begin{aligned}
\hat{\Phi}_{\varepsilon_{j}}\left(z_{j}\right)= & \hat{\Phi}_{\varepsilon_{j}}\left(v_{j}\right)-\Gamma_{V_{0} W_{0}}^{*}(u) \\
& +\int_{\mathbb{R}^{3}} \hat{W}_{\varepsilon_{j}}(x)\left(F\left(\left|v_{j}\right|\right)-F\left(\left|z_{j}\right|\right)-F\left(\left|\tilde{v}_{j}\right|\right)\right)+o(1) \\
= & o(1)
\end{aligned}
$$

as $j \rightarrow \infty$, which implies that $\hat{\Phi}_{\varepsilon_{j}}\left(z_{j}\right) \rightarrow 0$. Similarly, by $(5.16)$,

$$
\begin{aligned}
\hat{\Phi}_{\varepsilon}^{\prime}\left(z_{j}\right) \varphi & =\int_{\mathbb{R}^{3}} \hat{W}_{\varepsilon_{j}}(x)\left(f\left(\left|v_{j}\right|\right) v_{j}-f\left(\left|z_{j}\right|\right) z_{j}-f\left(\left|\tilde{v}_{j}\right|\right) \tilde{v}_{j}\right) \bar{\varphi}+o(1) \\
& =o(1)
\end{aligned}
$$

as $j \rightarrow \infty$ uniformly in $\|\varphi\| \leq 1$, which implies that $\hat{\Phi}_{\varepsilon_{j}}^{\prime}\left(z_{j}\right) \rightarrow 0$. Therefore,

$$
o(1)=\int_{\mathbb{R}^{3}} \hat{W}_{\varepsilon_{j}}(x) \bar{F}\left(\left|z_{j}\right|\right)
$$

This, together with $\left(g_{2}\right)$, shows

$$
\int_{\mathbb{R}^{3}} \hat{W}_{\varepsilon_{j}}(x) f\left(\left|z_{j}\right|\right)\left|z_{j}\right|^{2} \rightarrow 0
$$

Notice that $\left\{\left|z_{j}\right|_{\infty}\right\}$ is bounded so $\int_{\mathbb{R}^{3}} \hat{W}_{\varepsilon_{j}}(x) f\left(\left|z_{j}\right|\right)\left|z_{j}^{+}-z_{j}^{-}\right|^{2} \leq C^{2}$. As a consequence, we get

$$
\begin{aligned}
\left(1+\frac{\tau}{a}\right)\left\|z_{j}\right\|^{2} & \leq\left\|z_{j}\right\|^{2}+\Re \int_{\mathbb{R}^{3}} \hat{V}_{\varepsilon_{j}}(x)\left\langle z_{j}, z_{j}^{+}-z_{j}^{-}\right\rangle \\
& =\Phi_{\varepsilon_{j}}^{\prime}\left(z_{j}\right)\left(z_{j}^{+}-z_{j}^{-}\right)+\Re \int_{\mathbb{R}^{3}} \hat{W}_{\varepsilon_{j}}(x) f\left(\left|z_{j}\right|\right)\left\langle z_{j}, z_{j}^{+}-z_{j}^{-}\right\rangle \\
& \leq o(1)+C\left(\int_{\mathbb{R}^{3}} \hat{W}_{\varepsilon_{j}}(x) f\left(\left|z_{j}\right|\right)\left|z_{j}\right|^{2}\right)^{1 / 2} \\
& =o(1),
\end{aligned}
$$

that is, $\left\|z_{j}\right\| \rightarrow 0$ which, together with (5.14), yields $v_{j} \rightarrow u$ in $E$ as $j \rightarrow \infty$.

In order to verify that $v_{j} \rightarrow u$ in $H^{1}$, observe that by (5.11) and (5.12)

$$
A_{a} z_{j}=\hat{W}_{\varepsilon_{j}}(x) f\left(\left|v_{j}\right|\right) v_{j}-W_{0} f(|u|) u-\left(\hat{V}_{\varepsilon_{j}}(x) v_{j}-V_{0} u\right) .
$$

By the exponential decay of $u$ and the uniform estimate (3.6), it is easy to show that $\left|A_{a} z_{j}\right|_{2} \rightarrow 0$. Therefore $v_{j} \rightarrow u$ in $H^{1}$.

Step 2) $v_{j}(x) \rightarrow 0$ as $|x| \rightarrow \infty$ uniformly in $j \in \mathbb{N}$. Assume by contradiction that the conclusion does not hold. Then by the sub-solution estimate there exist $\sigma>0$ and $x_{j} \in \mathbb{R}^{3}$ with $\left|x_{j}\right| \rightarrow \infty$ such that $\sigma \leq\left|v_{j}\left(x_{j}\right)\right| \leq C_{0} \int_{B_{1}\left(x_{j}\right)}\left|v_{j}\right|$. Since $v_{j} \rightarrow u$ in $H^{1}$ one gets

$$
\begin{aligned}
\sigma & \leq C_{0} \int_{B_{1}\left(x_{j}\right)}\left|v_{j}\right| \leq C_{0} \int_{B_{1}\left(x_{j}\right)}\left|v_{j}-u\right|+C_{0} \int_{B_{1}\left(x_{j}\right)}|u| \\
& \leq C^{\prime}\left(\int_{\mathbb{R}^{3}}\left|v_{j}-u\right|^{2}\right)^{1 / 2}+C_{0} \int_{B_{1}\left(x_{j}\right)}|u| \rightarrow 0
\end{aligned}
$$


a contradiction.

Step 3) $\left\{\varepsilon_{j} y_{j}^{\prime}\right\}_{j}$ is bounded. Assume by contradiction that $\varepsilon_{j}\left|y_{j}^{\prime}\right| \rightarrow \infty$ (along a subsequence). Then $V_{0} \geq \tau_{\infty}$ and $W_{0}<\pi$ (by $\left(P_{1}\right)$ ) which, together with Lemma 4.9 (noting that $\gamma_{V_{0} W_{0}}^{*}$ attains), implies $\gamma_{V_{0} W_{0}}^{*}>\gamma_{\mu \pi}^{*}$ for any $\mu \in\left[\tau, \tau_{\infty}\right]$. On the other hand, choosing $\mu$ close to $\tau_{\infty}$ satisfying (5.3), it follows from (5.13) and Lemma $5.2, \lim _{\varepsilon_{j} \rightarrow 0} c_{\varepsilon_{j}}=\gamma_{V_{0} W_{0}}^{*}$ and $\lim _{\varepsilon_{j} \rightarrow 0} c_{\varepsilon_{j}} \leq \gamma_{\mu \pi}^{*}$, hence $\gamma_{V_{0} W_{0}}^{*} \leq \gamma_{\mu \pi}$, a contradiction. Therefore, we can assume $\varepsilon_{j} y_{j}^{\prime} \rightarrow y_{0}, V_{0}=V\left(y_{0}\right)$ and $W_{0}=W\left(y_{0}\right)$. So, $u(x)$ is a least energy solution of (5.9). Now by Step 2 it is easy to see that one may assume that $y_{j}=y_{j}^{\prime}$ is a maximum point of $\left|u_{j}\right|$.

Step 4) $\left\{\varepsilon y_{\varepsilon}\right\}_{\varepsilon}$ is bounded. Assume by contradiction that there is $\varepsilon_{j} \rightarrow 0$ with $\varepsilon_{j}\left|y_{j}\right| \rightarrow \infty$ where $y_{j}$ is a maximum point of $\left|u_{j}\right|\left(y_{j}=y_{\varepsilon_{j}}, u_{j}=u_{\varepsilon_{j}}\right)$. Repeating the above arguments one sees that any relative subsequence $y_{j}^{\prime}$ of $y_{j}$ and $v_{j}(x)=u_{j}\left(x+y_{j}^{\prime}\right)$ satisfies that $v_{j}(x) \rightarrow 0$ as $|x| \rightarrow \infty$ uniformly in $j \in \mathbb{N}$ and $\left\{\varepsilon_{j} y_{j}^{\prime}\right\}$ is bounded (Step 2), 3)). Consequently, $\varepsilon_{j}\left|y_{j}-y_{j}^{\prime}\right| \geq \varepsilon_{j}\left|y_{j}\right|-\varepsilon_{j}\left|y_{j}^{\prime}\right| \rightarrow \infty$, particularly, $\left|y_{j}-y_{j}^{\prime}\right| \rightarrow \infty$. Then, $\max \left|u_{j}\right|=\left|u_{j}\left(y_{j}\right)\right|=\left|v_{j}\left(y_{j}-y_{j}^{\prime}\right)\right| \rightarrow 0$, a contradiction.

Step 5) $\lim _{\varepsilon \rightarrow 0} \operatorname{dist}\left(\varepsilon y_{\varepsilon}, A_{w}\right)=0$. It is sufficient to check that $y_{0} \in \mathscr{A}_{w}$. By virtue of Proposition 4.1 and $\left(P_{3}\right), \gamma_{\tau \pi}^{*}$ is archived, it hence follows from (4.18) that

$$
\lim _{j \rightarrow \infty} c_{\varepsilon_{j}}=\lim _{j \rightarrow \infty} c_{\varepsilon_{j}}^{\tau \pi} \leq \gamma_{V^{\tau}(0) W^{\pi}(0)}^{*}=\gamma_{V(0) W(0)}^{*}=\gamma_{\kappa \pi}^{*}
$$

which, together with (5.13), shows

$$
\gamma_{V\left(y_{0}\right) W\left(y_{0}\right)}^{*} \leq \gamma_{\kappa \pi}^{*} .
$$

Since $\pi \geq W\left(y_{0}\right)$ one has $V\left(y_{0}\right) \leq \kappa$. If $\pi=W\left(y_{0}\right)$, i.e., $y_{0} \in \mathscr{W}$, there must be $V\left(y_{0}\right)=\kappa$ (because $\kappa=\min _{\mathscr{W}} V$ ). If $W\left(y_{0}\right)<\pi$ then we must have $V\left(y_{0}\right)<\kappa$. In conclusion, $y_{0} \in \mathscr{A}_{w}$.

The proof is hereby complete.

Lemma 5.5. There exists $C>0$ such that for all $j \in \mathbb{N}$

$$
\left|u_{j}(x)\right| \leq C e^{-\sqrt{\omega / 2}\left|x-y_{j}\right|} \quad \forall j \in \mathbb{N}
$$

where $\omega=a^{2}-\tau^{2}$

Proof. By the Step 2 of the proof of Lemma 5.4 we may take $\delta>0$ and $r>0$ such that $\left|v_{j}(x)\right| \leq \delta$ and

$$
\left|\Re\left[r_{\varepsilon_{j}}\left(x,\left|v_{j}\right|\right) v_{j} \frac{\overline{v_{j}}}{\left|v_{j}\right|}\right]\right| \leq \frac{\omega}{2}\left|v_{j}\right|
$$

for all $|x| \geq r, j \in \mathbb{N}$. This, together with (5.7), implies

$$
\Delta\left|v_{j}\right| \geq \frac{\omega}{2}\left|v_{j}\right| \text { for all }|x| \geq r, j \in \mathbb{N} .
$$

Let $\Gamma(y)=\Gamma(y, 0)$ be a fundamental solution to $-\Delta+\omega / 2$ (see, e.g., [27]). Using the uniform boundedness, one may choose $\Gamma$ so that $\left|v_{j}(y)\right| \leq \frac{\omega}{2} \Gamma(y)$ holds on $|y|=r$, all $j \in \mathbb{N}$. Let $z_{j}=\left|v_{j}\right|-\frac{\omega}{2} \Gamma$. Then

$$
\begin{aligned}
\Delta z_{j} & =\Delta\left|v_{j}\right|-\frac{\omega}{2} \Delta \Gamma \\
& \geq \frac{\omega}{2}\left(\left|v_{j}\right|-\frac{\omega}{2} \Gamma\right)=\frac{\omega}{2} z_{j} .
\end{aligned}
$$


By the maximum principle we can conclude that $z_{j}(y) \leq 0$ on $|y| \geq r$. It is well known that there is $C^{\prime}>0$ such that $\Gamma(y) \leq C^{\prime} \exp (-\sqrt{\omega / 2}|y|)$ on $|y| \geq 1$. We see that

$$
\left|v_{j}(y)\right| \leq C \exp (-\sqrt{\omega / 2}|y|)
$$

for all $y \in \mathbb{R}^{3}$ and all $j \in \mathbb{N}$, that is,

$$
\left|u_{j}(x)\right| \leq C \exp \left(-\sqrt{\omega / 2}\left|x-y_{j}\right|\right)
$$

for all $x \in \mathbb{R}^{3}$ and all $j \in \mathbb{N}$

The proof is completed.

Proof. [Proof of Theorem 2.2] Writing $\varepsilon=\varepsilon_{j}$ and going back to the equation (2.1) with the variable substitution $x \mapsto x / \varepsilon$, we set

$$
w_{\varepsilon}(x)=u_{\varepsilon}(x / \varepsilon) \quad \text { and } \quad x_{\varepsilon}=\varepsilon y_{\varepsilon} .
$$

Then $w_{\varepsilon}$ is a least energy solution of (2.1) for all $\varepsilon$ small, and $w_{\varepsilon} \in W^{1, s}$ for all $s \geq 2$ by Lemma 5.6. It is clear that $x_{\varepsilon}$ is a maximum point of $\left|w_{\varepsilon}\right|$, and the conclusions $\left(a_{1}\right)$ and $\left(a_{2}\right)$ follow from Lemma 5.5 and Lemma 5.4, respectively.

5.2. Proof of Theorem 2.3. Assume $\left(P_{0}\right)-\left(\hat{P}_{0}\right)$ and $\left(P_{2}\right)$ are satisfied. We can assume, without loss of generality, that $0 \in \mathscr{V}$ such that $W(0)=\max _{x \in \mathscr{V}} W(x)$. Then $V(0)=\tau$ and $\pi \geq \kappa:=W(0) \geq \pi_{\infty}$. Consider the functional $\Phi_{\varepsilon}$. We verify similarly to Lemma 5.1 the following existence.

Lemma 5.6. Let $\left(g_{1}\right)-\left(g_{2}\right),\left(P_{0}\right)-\left(\hat{P}_{0}\right)$ and $\left(P_{2}\right)$ be satisfied. Then $c_{\varepsilon}$ is attained for all small $\varepsilon>0$.

Proof. Given $\varepsilon>0$, let $u_{k} \in \mathscr{N}_{\varepsilon}$ be a minimizing sequence of $I_{\varepsilon}$ and set $w_{k}=$ $u_{k}+h_{\varepsilon}\left(u_{k}\right)$ which is a $(P S)_{c_{\varepsilon}}$ sequence for $\Phi_{\varepsilon}$ on $E$. We may assume $w_{k} \rightarrow w_{\varepsilon} \in \mathscr{K}_{\varepsilon}$ in $E$. If $w_{\varepsilon} \neq 0$ then clearly $\Phi_{\varepsilon}\left(w_{\varepsilon}\right)=c_{\varepsilon}$ and we are done.

Assume by contradiction that there is a sequence $\varepsilon_{j} \rightarrow 0$ with $w_{\varepsilon_{j}}=0$. Then $w_{k}=u_{k}+h_{\varepsilon_{j}}\left(u_{k}\right) \rightarrow 0$ in $E$ and $L^{s}$ for $s \in[2,3], w_{k} \rightarrow 0$ in $L_{l o c}^{s}$ for $s \in(1,3)$. and $w_{k}(x) \rightarrow 0$ a.e. for $x \in \mathbb{R}^{3}$. By $\left(\hat{P}_{0}\right)$, one can choose $\tau<\mu<\tau_{\infty}$ satisfying (5.3) for any $\nu \in\left[\pi_{\infty}, \pi\right]$. In particular, take $\nu=\kappa$ and consider the functional $\Phi_{\varepsilon_{j}}^{\mu \kappa}$. Since $V_{\varepsilon_{j}}^{\mu}(0) \leq \mu$ and $W_{\varepsilon_{j}}^{\kappa}(0)=\kappa$, and $m u$ and $\kappa$ satisfy (5.3), it follows from (4.19) that

$$
\lim _{j \rightarrow \infty} c_{\varepsilon_{j}}^{\mu \kappa}=\gamma_{\mu \kappa}^{*}
$$

As before, letting $t_{k}>0$ be such that $t_{k} u_{k} \in \mathscr{N}_{\varepsilon_{j}}^{\mu \kappa}$, and $O_{\varepsilon}:=\left\{x \in \mathbb{R}^{3}: V_{\varepsilon}(x) \leq\right.$ $\mu$ or $\left.W_{\varepsilon}(x)>\kappa\right\}$, we obtain

$$
\begin{aligned}
c_{\varepsilon_{j}}^{\mu \kappa} \leq & I_{\varepsilon_{j}}^{\mu \kappa}\left(t_{k} u_{k}\right) \leq I_{\varepsilon_{j}}\left(u_{k}\right)+\frac{1}{2} \int_{O_{\varepsilon_{j}}}\left(\mu-V_{\varepsilon_{j}}(x)\right)\left|t_{k} u_{k}+h_{\varepsilon_{j}}^{\mu \kappa}\left(t_{k} u_{k}\right)\right|^{2} \\
& +\int_{O_{\varepsilon_{j}}}\left(W_{\varepsilon_{j}}(x)-\kappa\right) F\left(\left|t_{k} u_{k}+h_{\varepsilon_{j}}^{\mu \kappa}\left(t_{k} u_{k}\right)\right|\right) \\
= & c_{\varepsilon_{j}}+o(1)
\end{aligned}
$$

as $k \rightarrow \infty$, hence, $c_{\varepsilon_{j}}^{\mu \kappa} \leq c_{\varepsilon_{j}}$, consequently,

$$
\gamma_{\mu \kappa}^{*} \leq \lim _{j \rightarrow \infty} c_{\varepsilon_{j}}
$$


On the other hand, let $v_{k} \in \mathscr{N}_{\varepsilon}^{\mu \kappa}$ be a minimizing sequence for $I_{\varepsilon}^{\mu \kappa}$ and set $E_{k}=$ $E^{-} \oplus \mathbb{R}^{+} v_{k}$. Since $V^{\mu} \geq V$ and $W^{\kappa} \leq W$, one has

$$
I_{\varepsilon}^{\mu \kappa}\left(v_{k}\right)=\max _{z \in E_{k}} \Phi_{\varepsilon}^{\mu \kappa}(z) \geq \max _{z \in E_{k}} \Phi_{\varepsilon}(z),
$$

so $c_{\varepsilon} \leq c_{\varepsilon}^{\mu \kappa}$. Therefore, the above discussion yields

$$
\gamma_{\mu \kappa}^{*}=\lim _{\varepsilon_{j} \rightarrow 0} c_{\varepsilon_{j}}^{\mu \kappa}=\lim _{\varepsilon_{j} \rightarrow 0} c_{\varepsilon_{j}} .
$$

In particular, for any $\tau<\mu_{1}<\mu_{2}<\tau_{\infty}$ so that (5.3) holds for $\mu=\mu_{1}$ and $\mu=\mu_{2}$, one obtains the following

$$
\gamma_{\mu_{1} \kappa}^{*}<\gamma_{\mu_{2} \kappa}^{*}=\lim _{\varepsilon_{j} \rightarrow 0} c_{\varepsilon_{j}}=\gamma_{\mu_{1} \kappa}^{*}
$$

a contradiction.

As a by-product of the above argument we have

Lemma 5.7. $\lim \sup _{\varepsilon \rightarrow 0} c_{\varepsilon} \leq \limsup _{\varepsilon \rightarrow 0} c_{\varepsilon}^{\mu \kappa}=\gamma_{\mu \kappa}^{*}$ for all $\mu$ satisfying (5.3).

We also point out that the above argument applies directly to verify that $\mathscr{S}_{\varepsilon}$ is compact for all small $\varepsilon>0$.

Now we study the concentration phenomenon under additionally $\left(P_{3}\right)$. Such an assumption implies particularly that $\gamma_{\tau \kappa}^{*}$ is archived.

Lemma 5.8. Assume additionally that $\left(P_{3}\right)$ is satisfied. Let $u_{\varepsilon} \in \mathscr{S}_{\varepsilon}$. There is a maximum point $y_{\varepsilon}$ of $\left|u_{\varepsilon}\right|$ such that $\lim _{\varepsilon \rightarrow 0} \operatorname{dist}\left(\varepsilon y_{\varepsilon}, \mathscr{A}_{v}\right)=0$, and for any sequence $\varepsilon y_{\varepsilon} \rightarrow y_{0}, v_{\varepsilon}(x):=u_{\varepsilon}\left(x+y_{\varepsilon}\right)$ converges in $H^{1}$ to a least energy solution of

$$
-i \alpha \cdot \nabla v+a \beta v+V\left(y_{0}\right) v=W\left(y_{0}\right) h(|v|) v .
$$

Proof. We are sketchy along the lines carried out previously for Lemma 5.4.

Step 1) Let $u_{j} \in \mathscr{S}_{j} \equiv \mathscr{S}_{\varepsilon_{j}}$ with $\varepsilon_{j} \rightarrow 0$. Then $\left\{u_{j}\right\}$ is bounded and nonvanishing. There exist a sequence $\left\{y_{j}^{\prime}\right\} \subset \mathbb{R}^{3}$ and constants $r>0, \delta>0$ such that

$$
\liminf _{j \rightarrow \infty} \int_{B_{r}\left(y_{j}^{\prime}\right)}\left|u_{j}\right|^{2} \geq \delta
$$

Then $v_{j}(x)=u_{j}\left(x+y_{j}^{\prime}\right)$ is a least energy solution of

$$
-i \alpha \cdot \nabla v_{j}+a \beta v_{j}+\hat{V}_{\varepsilon_{j}}(x) v_{j}=\hat{W}_{\varepsilon_{j}}(x) f\left(\left|v_{j}\right|\right) v_{j}
$$

where $\hat{V}_{\varepsilon_{j}}(x)=V\left(\varepsilon_{j}\left(x+y_{j}^{\prime}\right)\right)$ and $\hat{W}_{\varepsilon_{j}}(x)=W\left(\varepsilon_{j}\left(x+y_{j}^{\prime}\right)\right)$. The least energy

$$
\begin{aligned}
\hat{c}_{\varepsilon_{j}} & =\hat{\Phi}_{\varepsilon_{j}}\left(v_{j}\right)=\Phi_{\varepsilon_{j}}\left(u_{j}\right)=c_{\varepsilon_{j}} \\
& =\int_{\mathbb{R}^{3}} \hat{W}_{\varepsilon_{j}}(x) \bar{F}\left(\left|v_{j}\right|\right) .
\end{aligned}
$$

Moreover, $v_{j} \rightarrow u \neq 0$ in $E, v_{j} \rightarrow u$ in $L_{l o c}^{s}$ for $s \in[1,3)$, and $u$ solves

$$
-i \alpha \cdot \nabla u+a \beta u+V_{0} u=W_{0} f(|u|) u
$$


with the energy

$$
\Gamma_{V_{0} W_{0}}^{*}(u)=\int_{\mathbb{R}^{3}} W_{0} \bar{F}(|u|) \geq \gamma_{V_{0} W_{0}}^{*} .
$$

By the Fatou's lemma one gets

$$
\lim _{j \rightarrow \infty} c_{\varepsilon_{j}}=\Gamma_{V_{0} W_{0}}^{*}(u)=\gamma_{V_{0} W_{0}}^{*}
$$

and

$$
\lim _{j \rightarrow \infty} \int_{\mathbb{R}^{3}} \hat{W}_{\varepsilon_{j}}(x) \bar{F}\left(\left|v_{j}\right|\right)=\int_{\mathbb{R}^{3}} W_{0} \bar{F}(|u|)=\gamma_{V_{0} W_{0}}^{*} .
$$

Using these facts one sees as before that, in fact, $v_{j} \rightarrow u$ in $H^{1}$.

Step 2) $v_{j}(x) \rightarrow 0$ as $|x| \rightarrow \infty$ uniformly in $j \in \mathbb{N}$. This follows from (5.8) and the convergence of $v_{j} \rightarrow u$ in $H^{1}$.

Step 3) $\left\{\varepsilon_{j} y_{j}^{\prime}\right\}_{j}$ is bounded. Assume by contradiction that $\varepsilon_{j}\left|y_{j}^{\prime}\right| \rightarrow \infty$. Then $V_{0} \geq \tau_{\infty}>\tau$ and $W_{0} \leq \pi_{\infty} \leq \kappa$, which implies $\gamma_{V_{0} W_{0}}^{*} \geq \gamma_{\tau_{\infty} \pi_{\infty}}^{*}>\gamma_{\mu \pi_{\infty}}^{*} \geq \gamma_{\mu \kappa}^{*}$ for any $\mu \in\left[\tau, \tau_{\infty}\right)$. On the other hand, choosing $\mu<\tau_{\infty}$ satisfying (5.3), it follows from (5.17) and Lemma 5.7, that $\lim _{\varepsilon_{j} \rightarrow 0} c_{\varepsilon_{j}}=\gamma_{V_{0} W_{0}}^{*}$ and $\lim _{\varepsilon_{j} \rightarrow 0} c_{\varepsilon_{j}} \leq \gamma_{\mu \kappa}^{*}$, hence $\gamma_{V_{0} W_{0}}^{*} \leq \gamma_{\mu \kappa}^{*}$, a contradiction. Therefore, we can assume $\varepsilon_{j} y_{j}^{\prime} \rightarrow y_{0}, V_{0}=V\left(y_{0}\right)$ and $W_{0}=W\left(y_{0}\right)$. Now by Step 2 it is easy to see that one may assume that $y_{j}=y_{j}^{\prime}$ is a maximum point of $\left|u_{j}\right|$.

Step 4) $\left\{\varepsilon y_{\varepsilon}\right\}_{\varepsilon}$ is bounded.

Step 5) $\lim _{\varepsilon \rightarrow 0} \operatorname{dist}\left(\varepsilon y_{\varepsilon}, \mathscr{A}_{v}\right)=0$. It is sufficient to check that $y_{0} \in \mathscr{A}_{v}$. By $\left(P_{3}\right)$ and (4.18)

$$
\lim _{j \rightarrow \infty} c_{\varepsilon_{j}}=\lim _{j \rightarrow \infty} c_{\varepsilon_{j}}^{\tau \pi} \leq \gamma_{V^{\tau}(0) W^{\pi}(0)}^{*}=\gamma_{V(0) W(0)}^{*}=\gamma_{\tau \kappa}^{*}
$$

which, jointly with (5.17), implies

$$
\gamma_{V\left(y_{0}\right) W\left(y_{0}\right)}^{*} \leq \gamma_{\tau \kappa}^{*} .
$$

Since $V\left(y_{0}\right) \geq \tau$, one has $W\left(y_{0}\right) \geq \kappa$. If $V\left(y_{0}\right)=\kappa$, i.e. $y_{0} \in \mathscr{V}$, then $W\left(y_{0}\right)=\kappa$ because $\kappa=\max _{\mathscr{V}} W$. If $y_{0} \notin \mathscr{V}$ then there must be $W\left(y_{0}\right)>\kappa$. This proves that $y_{0} \in \mathscr{A}_{v}$.

The proof is hereby complete.

Now repeating the arguments of Lemma 5.5 we obtain

LEMma 5.9. There exists $C>0$ such that for all $\varepsilon$ small

$$
\left|u_{\varepsilon}(x)\right| \leq C e^{-\sqrt{\omega / 2}\left|x-y_{\varepsilon}\right|} \quad \forall x \in \mathbb{R}^{3} .
$$

Proof. [Proof of Theorem 2.3] Define

$$
w_{\varepsilon}(x)=u_{\varepsilon}(x / \varepsilon) \quad \text { and } \quad x_{\varepsilon}=\varepsilon y_{\varepsilon} .
$$

Then $w_{\varepsilon}$ is a least energy solution of (2.1) for all $\varepsilon$ small, $x_{\varepsilon}$ is a maximum point of $\left|w_{\varepsilon}\right|$, and the conclusion $\left(a_{1}\right)$ and $\left(a_{2}\right)$ follow from Lemma 5.9 and Lemma 5.8. 
5.3. Proof of Theorem 2.5. Proof. [Proof of Theorem 2.5] Consider the functional $\Phi_{\varepsilon}$.

Step 1. For each $\varepsilon>0$, there is $\delta_{\varepsilon}>0$ such that $\left\|u^{ \pm}\right\|^{2} \pm \int_{\mathbb{R}^{3}} V_{\varepsilon}(x)\left|u^{ \pm}\right|^{2} \geq$ $\delta_{\varepsilon}\left\|u^{ \pm}\right\|^{2}$. This implies that $\Phi_{\varepsilon}$ possesses the linking structure. Let $c_{\varepsilon}$ denote the linking level of $\Phi_{\varepsilon}$.

Step 2. There is $\varsigma>0$ independent of $\varepsilon$ such that $\Phi_{\varepsilon}$ satisfies $(P S)_{c}$ condition for all $c<\varsigma$. The proof is somewhat usual, see [16, Lemma 3.6] or [13] for example.

Step 3. Setting $V^{\mu}(x)=\max \{\mu, V(x)\}$ for $\mu \in\left(0, \tau_{\infty}\right), \nu=\inf W$, one has

$$
\begin{aligned}
\Phi_{\varepsilon}(u) & \leq \Phi_{\varepsilon}^{\mu}(u):=\frac{1}{2} \int_{\mathbb{R}^{3}}\left\langle\left(-i \alpha \cdot \nabla+a \beta+V_{\varepsilon}^{\mu}(x)\right) u, u\right\rangle-\int_{\mathbb{R}^{3}} W_{\varepsilon}(x) F(|u|) \\
& \leq \tilde{\Phi}_{\varepsilon}^{\mu}(u):=\frac{1}{2} \int_{\mathbb{R}^{3}}\left\langle\left(-i \alpha \cdot \nabla+a \beta+V_{\varepsilon}^{\mu}(x)\right) u, u\right\rangle-\nu c_{0} \int_{\mathbb{R}^{3}}|u|^{q}
\end{aligned}
$$

Let $c_{\varepsilon}^{\mu}$ and $\hat{c}_{\varepsilon}^{\mu}$ denote the linking levels respectively of $\Phi_{\varepsilon}^{\mu}$ and $\hat{\Phi}_{\varepsilon}^{\mu}$. The arguments of Lemma 4.10 are applicable (using (4.4) instead of (4.15)) to $\hat{\Phi}_{\varepsilon}^{\mu}$, which yields

$$
\limsup _{\varepsilon \rightarrow 0} \hat{c}_{\varepsilon}^{\mu} \leq \gamma_{\mu \nu}
$$

This, jointly with Lemma 4.6, implies that

$$
\limsup _{\varepsilon \rightarrow 0} \hat{c}_{\varepsilon}^{\mu} \leq(a+\mu)^{\frac{2(3-q)}{q-2}}\left(c_{0} \nu\right)^{\frac{-2}{q-2}} \gamma_{q}
$$

Thus

$$
\lim _{\varepsilon \rightarrow 0} \lim _{\mu \rightarrow-a} c_{\varepsilon}^{\mu}=0
$$

Take $\mu_{0}>-a$ such that $c_{\varepsilon}^{\mu_{0}}<\varsigma$ for all $\varepsilon$ small. Sice $c_{\varepsilon} \leq c_{\varepsilon}^{\mu}$, we see that, for all $\varepsilon$ small, (2.1) has a ground state solution $u_{\varepsilon}$ with $\Phi_{\varepsilon}\left(u_{\varepsilon}\right)=c_{\varepsilon} \rightarrow 0$ as $\varepsilon \rightarrow 0$.

Step 4. Finally, similarly to the last argument of the step 1 of Lemma 5.4, one checks that $u_{\varepsilon} \rightarrow 0$ in $H^{1}$. The proof is hereby complete.

Acknowledgments. The authors would like to thank the referees for the useful suggestions to improve the article.

\section{REFERENCES}

[1] Ackermann, N.: A nonlinear superposition principle and multibump solutions of peridic Schrödinger equations. J. Funct. Anal. 234, 423-443 (2006)

[2] Ackermann, N.: On a periodic Schrödinger equation with nonlocal superlinear part. Math. Z. 248, 423-443 (2004)

[3] Ambrosetti, A., Badiale, M., Cingolani, S.: Semiclassical states of nonlinear Schödinger equations. Arch. Rat. Mech. Anal. 140, 285-300 (1997)

[4] Ambrosetti, A., Malchiodi, A.: Perturbation Methods and Semilinear Elliptic Problems on $\mathbb{R}^{n}$. Birkhäuser Verlag, Basel, 2006

[5] Balabane, M., Cazenave, T., Douady, A., Merle, F.: Existence of excited states for a nonlinear Dirac field. Comm. Math. Phys. 119, 153-176 (1988)

[6] Bartsch, T., Ding, Y. H.: Deformation theorems on non-metrizable vector spaces and applications to critical point theory. Math. Nachr. 279, 1267-1289 (2006)

[7] Bartsch, T., Ding, Y. H.: Solutions of nonlinear Dirac equations. J. Differential Equations 226, 210-249 (2006) 
[8] Brezis, H.,Nirenberg, L.: Positive solutions of nonlinear elliptic equations involving critical Sobolev exponents. Comm. Pure Appl. Math. 36, 437-477 (1983).

[9] Byeon, J., Jeanjean, L.: Standing waves for nonlinear Schrödinger equations with a general nonlinearity. Arch. Ration. Mech. Anal. 185, 185-200 (2007)

[10] Coti-Zelati, V., Rabinowitz, P.: Homoclinic type solutions for a semilinear elliptic PDE on $\mathbb{R}^{n}$. Comm. Pure Appl. Math. 45, 1217-1269 (1992)

[11] Dautray, R., Lions, J. L.: Mathematical Analysis and Numerical Methods for Science and Technology. Vol. 3. Springer-Verlag, Berlin, 1990

[12] del Pino, M., Felmer, P.: Semi-classical states of nonlinear Schrödinger equations: a variational reduction method. Math. Ann. 324 1-32 (2002)

[13] Ding, Y. H.: Variational Methods for Strongly Indefinite Problems, Interdisciplinary Math. Sci. - Vol. 7, World Scientific Publ., 2007.

[14] Ding, Y. H.: Semi-classical ground states concerntrating on the nonlinear potential for a Dirac equation. J. Differential Equations 249, 1015-1034 (2010).

[15] Ding, Y. H., Ruf, B.: Solutions of a nonlinear Dirac equation with external fields. Arch. Ration. Mech. Anal. 190, 1007-1032 (2008)

[16] Ding, Y. H., Wei, J. C.: Stationary states of nonlinear Dirac equations with general potentials. Rev. Math. Phys. 20, (2008), 1007-1032.

[17] Esteban, M. J.; Séré, E. Stationary states of the nonlinear Dirac equation: a variational approach, Comm. Math. Phys. 171, 323-350(1995)

[18] Esteban, M. J., Lewin, M., Séré, E.: Variational methods in relativistic quantum mechanics. Bull. Amer. Math. Soc. 45, 535-593 (2008)

[19] Floer, A., Weinstein, A.: Nonspreading wave packets for the cubic Schrödinger equation with a bounded potential. J. Funct. Anal. 69, 397-408 (1986)

[20] Gilbarg, D., Trudinger, N. S.: Elliptic Partial Differential Equations of Second Order. Springer, Berlin, 1983.

[21] Lions, P.L., The concentration-compactness principle in t he calculus of variations. The locally compact case. Part II. Ann. Inst. H. Poincaré, Non linéaire 1 (1984), 223-283.

[22] Merle, F.: Existence of stationary states for nonlinear Dirac equations. J. Differential Equations 74, 50-68 (1988)

[23] Pankov, A.: Periodic nonlinear Schrödinger equations with application to photonic crystals. Milan J. Math. 73, 259-287 (2005)

[24] Rabinowitz, Paul H.: On a class of nonlinear Schrödinger equations. Z. Angew Math. Phys. 43, 270-291 (1992)

[25] Ranada, A. F.: Classical Nonlinear Dirac Field Mmodels of Extended Particles. In Quantum Theory, Groups, Fields and Particles (ed. Barut, A.O.). Reidel, Amsterdam, 1982

[26] Reed, M., Simon, B.: Methods of Mathematical Physics, vols. I-IV, Academic Press, 1978.

[27] Simon, B.: Schrödinger semigroups. Bull. Amer. Math. Soc. (N.S.) 7, 447-526 (1982)

[28] Szulkin, A., Weth, T.: Ground state solutions for some indefinite problems. J. Funct. Anal. 257, 3802-3822 (2009)

[29] Thaller, B.: The Dirac Equation. Texts and Monographs in Physics, Springer, Berlin, 1992

[30] Willem, M.: Minimax Theorems. Birkhäuser, 1996.

[31] Wang, X. F.: One concentration of positive bound states of nonlinear Schrödinger equations. Comm. Math. Phys. 153, 229-244 (1993) 